Working Paper

Economic Series 10 - 40

December 2010
Departamento de Economía

Universidad Carlos III de Madrid

Calle Madrid, 126

28903 Getafe (Spain)

Fax (34) 916249875

\title{
“AVERAGE-BASED VERSUS HIGH- AND LOW-IMPACT INDICATORS FOR THE EVALUATION OF SCIENTIFIC DISTRIBUTIONS”
}

\author{
Pedro Albarrán ${ }^{a}$, Ignacio Ortuño ${ }^{b}$, Javier Ruiz-Castillo ${ }^{b}$ \\ ${ }^{a}$ Departamento de Fundamentos del Análisis Económico, Universidad de Alicante \\ ${ }^{\mathrm{b}}$ Departamento de Economía, Universidad Carlos III, and Research Associate of the CEPR \\ Project SCIFI-GLOW
}

\begin{abstract}
Albarrán et al. (2011a) introduced a novel methodology for the evaluation of citation distributions consisting of a pair of high- and a low-impact measures defined over the set of articles with citations below or above a critical citation level CCL. Albarrán et al. (2011b) presented the first empirical applications to a situation in which the world citation distribution in 22 scientific fields is partitioned into three geographical areas: the U.S., the European Union, and the rest of the world. In this paper, we compare our results with those obtained with average-based indicators. For reasonable CCLs, such as the $80^{\text {th }}$ percentile of the world citation distribution in each field, the cardinal differences between the results obtained with our highimpact index and the mean citation rate are of a large order of magnitude. When, in addition, the percentage in the top $5 \%$ of most cited articles or the percentage of uncited articles are used, there are still important quantitative differences with respect to the high- and low-impact indicators advocated in our approach when the CCL is fixed at the $80^{\text {th }}$ or the $95^{\text {th }}$ percentile.
\end{abstract}

\section{Acknowledgements}

This is the second version of a paper that appeared as Working Paper 10-10, Universidad Carlos III, May 2009. The authors acknowledge financial support from the Santander Universities Global Division of Banco Santander. Albarrán and Ruiz-Castillo also acknowledge financial help from the Spanish MEC, the first through grants SEJ2007-63098 and SEJ2006-05710, and the second through grant SEJ2007-67436. This paper is part of the SCIFI-GLOW Collaborative Project supported by the European Commission's Seventh Research Framework Programme, Contract number SSH7-CT-2008-217436. Comments and suggestions by two referees have contributed to improving the final version of the paper. 


\section{INTRODUCTION}

Albarrán et al. (2009a) introduced a novel methodology for the evaluation of research units of a certain size working in the same homogeneous field, namely, a scientific field where the number of citations received by any two papers is comparable independently of the journal where they have been published. The methodology is based on three points. Firstly, since citation distributions are highly skewed, their upper and lower parts are typically very different. ${ }^{1}$ Consequently, it seems useful to describe a distribution by means of two real valued functions defined over the subsets of articles with citations above or below a critical citation line. These will be referred to as a high-and a low-impact indicator, respectively. Secondly, a citation distribution can be identified with an income distribution: instead of individuals we have scientific articles, and instead of dollars we have citations. Once this step is taken, it can be seen that the measurement of low-impact coincides with the measurement of economic poverty, where the poor are defined as those individuals whose incomes are below a certain poverty line (which plays an analogous role to the critical citation line for a citation distribution). In turn, it is equally natural to identify the measurement of high-impact with the measurement of a certain notion of economic affluence. Thirdly, the question of which low-impact indicators might be used in practice can be answered in terms of a family of indices -originally suggested by Foster et al. (1984)- that satisfies a number of desirable properties, and has been widely used for the measurement of economic poverty in the last 25 years. These same properties lead to the selection of an equally convenient class of high-impact measures.

\footnotetext{
${ }^{1}$ See inter alia Seglen (1992), Shubert et al. (1987) for evidence concerning scientific articles published in the period 1981-85 in 114 sub-fields, Glänzel (2007) for articles published in 1980 in 12 broad fields and 60 middlesized disciplines, Albarrán and Ruiz-Castillo (2009) for articles published in the period 1998-2002 in 22 broad fields, and Albarrán et al. (2010a) for these same articles classified in $219 \mathrm{Web}$ of Science categories and three aggregation schemes consisting of a number of disciplines and broad fields.
} 
For this methodology to be of interest in bibliometrics, we must demonstrate that it is useful in practice. Albarrán et al. (2011b) presented the first empirical application of this approach using a large dataset acquired from Thomson Scientific for the evaluation of the citation impact in three geographical areas: the U.S.; the EU, namely, the 15 countries forming the European Union before the 2004 accession, and the remaining countries grouped in an area referred to as the rest of the world (RW hereafter). In this paper we complete the illustrative nature of Albarrán et al. (2011b) with a comparison of some of its main results with those that can be obtained using average-based and other indicators of scientific performance for the same dataset and the same partition of the world. ${ }^{2}$

The only information required from research units in the approach advocated in Albarrán et al. (2011a, b) is the homogeneous field to which each unit's publications belong and the number of citations each paper receives. In this scenario, the mean citation rate (MCR hereafter) is a good overall indicator of scientific performance. Consequently, the first empirical exercise in this paper compares the results obtained in Albarrán et al. (2011b) with those obtained exclusively using the MCR. Ordinal and cardinal issues are typically raised. From an ordinal point of view, two questions are asked. (i) Within each homogeneous field, does it always follow that when a geographical area has a greater MCR than another, the former has also a greater high-impact and a smaller low-impact level than the latter? (ii) Within a given geographical area, does the ranking of fields induced by a normalized MCR coincide with the ranking induced by a high- or a low-impact indicator? From a cardinal point of view, the typical issue refers to the extent to which one geographical area dominates another in a given homogeneous field. For example, the

\footnotetext{
${ }^{2}$ The relationship between high- and low-impact levels and publication shares across geographical areas in each field, and publication efforts across fields in each area, as well as the impact of international coauthorship on each geographical area's scientific performance are topics tackled in Albarrán et al. (2011b) that are not studied here at all. The impact of changes in critical citation levels is briefly mentioned in Section III.
} 
question is: by how much does the U.S. dominate the EU in Physics according to a highimpact indicator and according to the MCR?

It must be recognized that a single statistic of centrality -such as the MCR or the median- may not adequately summarize the asymmetries presented by a typically skewed citation distribution (see inter alia Bornmann et al., 2008 and, in a different context, Glänzel, 2002). In particular, authors from the Leiden group are very aware of the need to include in their battery of indicators some that capture what takes place at the tails of any citation distribution. Thus, together with average-based indicators, since its inception this group has always taken into account the papers published by a research unit that have received no citations at all (see, inter alia Moed et al., 1985, 1988, 1995, and van Raan, 2004). More recently, the Leiden group has turned its attention to the upper tail of the distribution, and has introduced the percentage in the top $5 \%$ of the most highly cited papers as an indicator of scientific excellence (see Tijssen et al., 2002, and van Leeuwen et al., 2003, as well as Aksnes and Sivertsen, 2004). In this paper we will refer to the MCR and these two indicators as the Leiden triad. In the second empirical exercise of this paper the results in Albarrán et al. (2011b) for high- and low-impact indicators are compared with the percentage of articles in the top 5\% of highly cited papers, and the percentage of articles with no citations at all, respectively. As before, ordinal and cardinal comparisons are performed. ${ }^{3}$

\footnotetext{
${ }^{3}$ It should be emphasized that, contrary to what is assumed in this paper, members of the Leiden group usually define their measures relying on information about the journal where each paper is published. This information allows them to compare the observed behavior of relatively small research units, namely research groups, with the expected behavior of the set of journals where the research group is known to publish. The ratio of such expected behavior to the behavior of the journals in the entire field constitutes another interesting indicator in this case. Finally, the possibility of ordering the set of journals in a field in terms of their relative impact allows authors in the Budapest group to graphically represent relative impact indicators in two-dimensional relational charts (Schubert and Braun, 1986, and Glänzel et al., 2002).
} 
Our choice of a homogeneous field should be clarified at the outset. Naturally, the smaller the set of closely linked journals used to define a given research field, the greater the homogeneity of citation patterns among the articles included must be. Therefore, ideally one should always work at the lowest aggregation level that the data allows. In our case, this may mean the 219 Web of Science categories distinguished by Thomson Scientific. However, articles are assigned to Web of Science categories through the assignment of the journals where they have been published. Many journals are unambiguously assigned to one specific category, but many other typically receive a multiple assignment. As a result, only about $58 \%$ of the total number of articles published in 1998-2007 is assigned to a single Web of Science category (see Albarrán et al., 2010a). On the other hand, Thomson Scientific distinguishes between 20 broad fields for the natural sciences and two for the social sciences. Although this firm does not provide a link between the 219 Web of Science categories and the 22 broad fields, Thomson Scientific assigns each article in our dataset to a single broad field. Therefore, given the illustrative nature of our work at this point, both in Albarrán et al. (2011b) and in this paper a homogeneous field is identified with one of the 22 Thomson Scientific broad fields. In this way, the thorny problems raised by the multiple assignments of articles to Web of Science categories, as well as the difficulties involved in the aggregation from the Web of Science to the broad field level, are provisionally avoided.

The rest of this paper is organized into three Sections. Section II introduces the indicators and the methods that will be used in the empirical part of the paper. Section III presents the data and the empirical findings, while Section IV discusses the results and offers some conclusions and extensions. 


\section{NOTATION, DEFINITIONS AND METHODS}

\section{1. Notation}

A discrete citation distribution of papers published in a given year is an ordered, nonnegative vector $x=\left(x_{1}, \ldots, x_{i}, \ldots, x_{n}\right)$, where $x_{1} \leq x_{2} \leq \ldots \leq x_{n}$, and $x_{i} \geq 0$ is the number of citations received by the $i$-th article over a certain number of years since its publication date -a period known as the citation window. Given a distribution $x$ and a critical citation line, $z \geq 0$, classify as low- or high-impact articles all papers with citation $x_{i} \leq z$, or $x_{i}>z$. Denote by $n(x)$ the total number of articles in the distribution, and by $l(x ; z)$ and $h(x ; z)=$ $n(x)-l(x ; z)$ the number of low- and high-impact articles. A low-impact index is a real valued function $L$ whose typical value $L(x ; z)$ indicates the low-impact level associated with the distribution $x$ and the critical citation line $z$, while a bigh-impact index is a real valued function $H$ whose typical value $H(x ; z)$ indicates the high-impact level associated with the distribution $x$ and the critical citation line $z$. We say that a high-impact (low-impact) measure is monotonic if one more citation increases (decreases) its value.

\section{2. The Leiden Triad of Indicators}

Given a citation distribution $x$, and a critical citation line $z=95^{\text {th }}$ percentile of the world citation distribution in that field, the high-impact index

$$
H\left(x ; z=95^{t h}\right)=h\left(x ; z=95^{t h}\right) / n(x)
$$

is the percentage of articles in distribution $x$ belonging to the top $5 \%$ of highly cited articles in the world. Similarly, given a citation distribution $x$, and a critical citation line $z=0$, the low-impact index

$$
L(x ; z=0)=b(x ; z=0) / n(x)
$$

is the percentage of articles without citations in that distribution. 
It is important to understand that, not being monotonic, the mere percentage of articles satisfying some interesting condition -such as the indices defined in (1) and (2)only captures what can be referred to as the incidence aspect of the phenomenon in question. ${ }^{4}$ To appreciate this limitation, consider a numerical example where $z$ is four, and there are two equal sized citation distributions with the same number of high-impact articles: $(5,6,7)$ for distribution $A$ and $(9,10,15)$ for distribution $B$. A measure of highimpact that only takes into account the incidence aspect of that phenomenon will not distinguish between the two research units. For example, in the situation just described $H(A ; z=4)=H(B ; z=4)$ according to the index defined in equation 1 . However, a monotonic high-impact measure that takes into account not only the incidence but also the intensity aspect of this phenomenon would $\operatorname{rank} A$ below $B$. A similar example would show the analogous limitations of the low-impact index $L(x ; z=0)$ introduced in equation 2.

Consider instead the MCR of the distribution $x, \mu(x)$, defined as

$$
\mu(x)=\left(\sum_{i} x_{i}\right) / n(x) .
$$

Since the MCR increases with the percentage and the total citations received by highimpact articles, it is sensitive to the incidence and the intensity aspects of a citation distribution. However, to see the limitations of indicators that only capture these two dimensions consider two research units $\mathrm{C}$ and $\mathrm{D}$ that are equally ranked in terms of both aspects of the high-impact phenomenon, and assume that most of the highly cited papers of unit $\mathrm{C}$ have a similar number of citations above the critical citation line, while a large part

\footnotetext{
${ }^{4}$ This is also the only aspect considered in Albarrán et al. (2010b), where the U.S. and the EU performance were compared using a variety of high-impact indices $H(x ; z)=h(x ; z) / n(x)$ for a number of critical citation lines fixed at different percentiles of the world citation distribution for the 22 broad fields distinguished by Thomson Scientific.
} 
of the citations received by unit $\mathrm{D}$ are concentrated in a few articles of, say, Nobel prize quality. In such situations it might be desirable that a high-impact measure takes into account the citation inequality among the set of high-impact papers, in which case unit D will exhibit a greater high-impact level than unit C. Of course, the MCR fails to respond to distributional changes at either tail of a distribution that maintains the MCR constant. ${ }^{5}$

\section{3. The FGT Family of Low- and High-impact Indicators}

Given a citation distribution $x$ and a critical citation line $z$, the Foster, Greer and Thorbecke (FGT hereafter) family of low-impact indicators, originally introduced in Foster et al. (1984) for the measurement of economic poverty, is defined by:

$$
L_{\beta}(x ; z)=[1 / n(x)] \Sigma_{i}=1^{l(x ; z)}\left(\Gamma_{i}\right)^{\beta}, 0 \leq \beta,
$$

where $\beta$ is a parameter identifying the members of the family, $\Gamma_{i}=\left(z-x_{i}\right) / z$ is the normalized low-impact gap for any article with $x_{i}$ citations. Observe that $\Gamma_{i} \geq 0$ for lowimpact articles, while $\Gamma_{i}=0$ for high-impact articles. The class of FGT high-impact indicators is defined by

$$
H_{\beta}(x ; z)=[1 / n(x)] \Sigma_{i}=l(x ; z)+1^{n(x)}\left(\Gamma^{*}\right)^{\beta}, 0 \leq \beta,
$$

where $\beta$ is again a parameter identifying the members of the family, $\Gamma^{*}{ }_{i}=\left(x_{i}-z\right) / z$ is the normalized high-impact gap. In this case, $\Gamma^{*}{ }_{i}>0$ for high-impact articles, while $\Gamma^{*_{i}}=0$ for low-impact articles.

It will be sufficient to understand the differences involved in the use of members of these two classes for parameter values $\beta=0,1$, and 2. Firstly, note that the high- and low-

\footnotetext{
${ }^{5}$ For a numerical example, assume that the critical citation line is four, and consider two equal sized citation distributions $\mathrm{C}$ and $\mathrm{D}$ with the same number of high-impact articles. Let $(10,10)$ and $(5,15)$ be the sets of high-impact articles with the same MCR equal to 10. A reasonable high-impact indicator sensitive to distributional considerations would rank unit $\mathrm{D}$ strictly above unit $\mathrm{C}$.
} 
impact indices obtained when $\beta=0$ coincides with the proportion of high- or low-impact papers:

$$
H_{d}(x ; z)=h(x ; z) / n(x)
$$

and

$$
L_{d}(x ; z)=l(x ; z) / n(x)
$$

Of course, $H_{0}(x ; z)+L o(x ; z)=1$, so that if $H_{0}(x ; z)$ changes, then $L o(x ; z)$ must change in the opposite direction. It should be noted that both the high- and low-impact indicators $H_{0}$ and Lo only capture the incidence aspect of both phenomena. Secondly, consider the highimpact index corresponding to the parameter value $\beta=1$, or the per-article bigh-impact gap ratio:

$$
H_{1}(x ; z)=[1 / n(x)] \sum_{i}=l(x ; z)+1^{n(x)} \Gamma^{*} i
$$

Similarly, the member of the FGT family of low-impact indicators for $\beta=1$, or the perarticle low-impact gap ratio, is equal to:

$$
L_{1}(x ; z)=[1 / n(x)]\left[\sum_{i=1} 1^{l(x ; z)} \Gamma i\right] .
$$

Both $H_{1}$ and $L_{1}$ are seen to capture both the incidence and the intensity of these phenomena. Thirdly, the high- and low-impact members of the FGT families obtained when $\beta=2$ can be expressed as:

$$
\begin{aligned}
& H_{2}(x ; z)=H_{0}(x ; z)\left\{\left[\left(H_{1}(x ; z)\right]^{2}+\left[1-H_{1}(x ; z)\right]^{2}(\mathrm{CH})^{2}\right]\right\}, \\
& L_{2}(x ; z)=L_{0}(x ; z)\left\{\left[\left(L_{0}(x ; z)\right]^{2}+\left[1-L_{1}(x ; z)\right]^{2}\left(\mathrm{CL}^{2}\right]\right\},\right.
\end{aligned}
$$

where $(\mathrm{CH})^{2}$ and $(\mathrm{CL})^{2}$ are the squared coefficient of variation (that is, the ratio of the standard deviation over the mean) among the high- and low-impact articles, respectively. In so far as the coefficients of variation $\mathrm{C}_{\mathrm{H}}$ and $\mathrm{C}_{\mathrm{L}}$ are two measures of citation inequality, the FGT indicators $H_{2}$ and $L_{2}$ defined in equations 8 and 9 simultaneously cover the incidence, 
the intensity, and the citation inequality aspects of the high- and low-impact phenomenon they measure. ${ }^{6}$

\section{4. Methods}

As indicated in the Introduction, we distinguish between ordinal and cardinal comparisons.

\section{$\underline{\text { Ordinal Comparisons }}$}

Among ordinal comparisons, the following two will be first examined. Firstly, we say that the MCR ranks scientific performance across geographical areas in a given field in the same way as indicators $H_{\beta}$ and $H_{\beta}$ for some parameter value $\beta$ and CCL $z$, if for any two areas $k$ and $l$ in that field we have that

$$
\mu\left(x^{k}\right)>\mu\left(x^{l}\right) \Rightarrow H_{\beta}\left(x^{k}, z\right)>H_{\beta}\left(x^{l}, z\right) \text { and } L_{\beta}\left(x^{k}, z\right)<L_{\beta}\left(x^{l}, z\right)
$$

That is, if area $k$ has a greater MCR, then it must have a greater high-impact and a smaller low-impact level than area $l$. Secondly, it is also interesting to compare the ranking of fields in a geographical area according to our indicators and the MCR. Therefore, we say that the ratio $\mu\left(x^{k}\right) / \mu(x)$ ranks scientific performance across geographical areas in a given field in the same way as indicators $H_{\beta}$ and $H_{\beta}$ for some parameter value $\beta$, if for some fields $i$ and $j$ and

CCLs $z i$ and $z j$ we have that

$$
\begin{aligned}
& \mu\left(x_{i}^{k}\right) / \mu\left(x_{i}\right)>\mu\left(x_{j}^{k}\right) / \mu\left(x_{j}\right) \Rightarrow \\
& H_{\beta}\left(x_{i}^{k} ; z i\right)>H_{\beta}\left(x_{j}^{k} ; z_{j}\right) \text { and } L_{\beta}\left(x_{i}^{k} ; z_{i}\right)<L_{\beta}\left(x_{j}^{k} ; z_{j}\right) .
\end{aligned}
$$

\footnotetext{
${ }^{6}$ Albarrán et al. (2011a) contains a full discussion of all the properties possessed by a number of approaches to the evaluation of research units, including all indicators presented in Sections II.2 and II.3.
} 
That is, if area $k$ 's relative contribution to the world MCR in field $i$ is greater than in field $j$, then it must have a greater high-impact and a smaller low-impact level in field $i$ than in field $j$.

Similarly, we say that the percentage of articles in the top 5\% ranks bigh-impact performance across geographical areas in a given field as $H_{\beta}$ for some parameter value $\beta$ and $C C L z$, if for any two areas $k$ and $l$ in that field we have that

$$
H\left(x^{k} ; z=95^{t h}\right)>H\left(x^{l} ; z=95^{t h}\right) \Rightarrow H_{\beta}\left(x^{k}, z\right)>H_{\beta}\left(x^{l}, z\right) .
$$

Next, for ordinal comparisons across fields $i$ and $j$ in a given area $k$, we say that the percentage of articles in the top $5 \%$ in area $k$ ranks high-impact performance across fields as $H_{\beta}$ for some parameter value $\beta$ if for some fields $i$ and $j$ and CCLs $z i$ and $z j$ we have that

$$
H\left(x_{i}^{k} ; z=95^{t b}\right)>H\left(x_{j}^{l} ; z=95^{t b}\right) \Rightarrow H_{\beta}\left(x_{i}^{k} ; z i\right)>H_{\beta}\left(x_{j}^{k} ; z j\right) .
$$

As far as the low-impact phenomenon is concerned, we begin by saying that the percentage of uncited articles ranks low-impact performance across geographical areas in a given field as $L_{\beta}$ for some parameter value $\beta$ and CCL $z$ if for any two areas $k$ and $l$ in that field we have that

$$
L\left(x^{k} ; z=0\right)>L\left(x^{l} ; z=0\right) \Rightarrow L_{\beta}\left(x^{k}, z\right)>L_{\beta}\left(x^{l}, z\right) .
$$

Finally, for ordinal comparisons across fields we say that the ratio $p o\left(x^{k}\right) / p o(x)$ ranks lowimpact performance across fields as $L_{\beta}$ for some parameter value $\beta$ if for some fields $I$ and $j$ and CCLs $z i$ and $z j$ we have that

$$
L\left(x^{k} ; z=0\right)>L\left(x^{l} ; z=0\right) \Rightarrow L_{\beta}\left(x_{i}^{k} ; z i\right)<L_{\beta}\left(x_{j}^{k} ; z j\right)
$$

\section{Cardinal Comparisons}


As far as cardinal comparisons are concerned, recall from Albarrán et al. (2011a) that the FGT family of high-impact indicators is decomposable in the sense that, given a critical citation line $z$, a parameter value $\beta$, and any partition of a citation distribution $x=\left(x^{1}, \ldots\right.$, $\left.x^{K}\right)$ into $K$ geographical areas, indexed by $k=1, \ldots, K$, the overall high-impact measure for $x$ can be expressed as:

$$
H_{\beta}(x ; z)=\Sigma k \omega k H_{\beta}\left(x^{k} ; z\right)
$$

where $H_{\beta}\left(x^{k} ; z\right)$ is the high-impact index value for geographical area $k$, and $\omega k$ is the area's publication share in distribution $x$. Similarly, the overall low-impact measure can be expressed as:

$$
L_{\beta}(x ; z)=\Sigma k \omega k L_{\beta}\left(x^{k} ; z\right),
$$

where $L_{\beta}\left(x^{k} ; z\right)$ is the low-impact index value for area $k$. To adequately interpret the results below, it is important to make it explicit that, from a normative point of view, for any geographical area $k$ it is preferable to have a high $H_{\beta}\left(x^{k} ; z\right)$ and a low $L_{\beta}\left(x^{k} ; z\right)$.

In order to quantify the relative situation of any geographical area in a given field, it is convenient to refer to the ratio $\omega k H_{\beta}\left(x^{k} ; z\right) / H_{\beta}(x ; z)$ as area $k$ 's observed contribution (OC hereafter) relative to the overall high-impact level for that $\beta$. We may ask: what is this area's relative expected contribution (EC hereafter) to that level? Clearly, the answer is its publication share $\omega k$. Thus, the ratio $\mathrm{OC} / \mathrm{EC}=H_{\beta}\left(x^{k} ; z\right) / H_{\beta}(x ; z)$ is greater than, equal to, or smaller than one as area k's OC is greater than, equal to, or smaller than this area's EC, namely its publication share $\omega k$. Similarly, the ratio $L_{\beta}\left(x^{k} ; z\right) / L_{\beta}(x ; z)$ is greater than, equal to, or smaller than one as area $k$ 's $\mathrm{OC}$ is greater than, equal to, or smaller than area $k$ 's $\mathrm{EC}$, or $\omega k$. 
All indicators in the Leiden triad defined in equations 1, 2, and 3 are also decomposable. For example, for the MCR we have:

$$
\mu(x)=\Sigma_{k} \omega k \mu\left(x^{k}\right)
$$

where $\mu\left(x^{k}\right)$ is the MCR in geographical area $k$. Note that the observed contribution of any area $k$ relative to the overall level, OC, is equal to the share of total citations, $s\left(x^{k}\right)$ :

$$
\mathrm{OC}=\omega k \mu\left(x^{k}\right) / \mu(x)=\left(\sum_{i} x_{i}^{k}\right) /\left(\sum_{k} \sum_{i} x_{i}^{k}\right)=s\left(x^{k}\right)
$$

Thus, given that the expected contribution of any geographical area, EC, is again its publication share, $\omega k$, the ratio

$$
\mathrm{OC} / \mathrm{EC}=\mu\left(x^{k}\right) / \mu(x)=s\left(x^{k}\right) / \omega k
$$

is greater than, equal to, or smaller than one as the citation share $s\left(x^{k}\right)$ is greater than, equal to, or smaller than this area's publication share $\omega k$. Similarly, for the indicators $H(x ; z=$ $\left.95^{t b}\right)$ [and $L(x ; z=0)$ ], we have that for any geographical area the ratio of the observed to the expected contribution is greater than, equal to, or smaller than one as the area's share in the top 5\% highly cited articles [or the set of uncited articles] in the world is greater than, equal to, or smaller than the area's publication share $\omega k$.

\section{EMPIRICAL RESULTS}

\section{1. The Data}

In this paper, only research articles or, simply, articles are studied. For reasons explained in the Introduction, the key assumption that permits the link between theoretical concepts and the data is the identification of homogeneous fields with the 20 natural sciences and the two social sciences distinguished by Thomson Scientific. After the elimination of observations with missing values for some variables, this paper refers to 
3,654,675 articles published in the period 1998-2002 with a five-year citation window. The total number of citations amount to $28,296,113$. Articles are assigned to geographical areas according to the institutional affiliation of their authors as recorded in the Thomson Scientific database on the basis of what has been indicated in the by-line of the publications. In any field, an article might be written by one or more scientists working in only one of the three geographical areas, or it might be co-authored by scientists working in two or three of them. In every internationally co-authored article a whole count is credited to each contributing area. ${ }^{7}$

The information about the ratios $H_{\beta}\left(x^{k} ; z\right) / H_{\beta}(x ; z)$ and $L_{\beta}\left(x^{k} ; z\right) / L_{\beta}(x ; z)$ for every $k$, every field, and every $\beta$ in the two FGT families of high- and low-impact indicators when the CCL is equal to the $80^{\text {th }}$ percentile is in Table 1 (which reproduces Table 4 in Albarrán et al., 2009b). The information for every $k$ and every field about the ratios $\mu\left(x^{k}\right) / \mu(x), H\left(x_{i}^{k}\right.$; $\left.z=95^{\text {th }}\right)$ and $L\left(x^{k} ; z=0\right)$ that we associate with the Leiden triad is in Table 2.

Tables 1 and 2 around here

\section{2. A Comparison of FGT Indicators and MCRs}

As indicated in the Introduction, the empirical part of this paper consists of two exercises. The results obtained in Albarrán et al. (2011b) will be first compared with those obtained using only the MCR. We distinguish between ordinal and cardinal comparisons. Among ordinal comparisons, we presently test if expression (10) is the case for parameter values $\beta=0,1,2$ when the CCL is fixed in each field at the $80^{\text {th }}$ percentile of the world citation distribution. In columns 1 to 3 in Table 2 it is observed that the ranking of areas

\footnotetext{
${ }^{7}$ See Albarrán et al. (2011b) for some descriptive statistics about the number of articles and publication shares by authorship type (Table 1), the classification of articles by scientific field and geographical area (Table 2), the absolute number of citations at the critical citation line in every field, the multiple of the mean that this number represents, and the percentage of the total number of citations received by the high-impact articles in each case (Table 3).
} 
according to the MCR is always the same in all fields ${ }^{8}$ : the U.S. above the EU, and the latter above the RW. However, as was seen in Section 4.1 in Albarrán et al. (2011b), when the CCL is fixed at the $80^{\text {th }}$ percentile and all values of $\beta=0,1,2$ are considered, the implication (10) is not satisfied on two occasions: in Immunology the EU has a lower highimpact level than the RW according to $H_{2}$, and in Engineering the U.S. has a greater lowimpact level than the EU according to $L_{2}$ (the Immunology and Engineering cases are illustrated in Figures 1 and 3 below). Since both cases arise for indicators responsive to distributional considerations, it is not surprising that the MCR approach fails to register the same order. It can be concluded that, except for these two instances, when there are only three areas under contention the MCR behaves as an excellent ordinal indicator of scientific performance.

Secondly, it is also interesting to compare the ranking of fields in a geographical area according to our indicators and the MCR. Given a CCL equal to the $80^{\text {th }}$ percentile of the corresponding world citation distribution, let us choose one member of the two FGT families of indicators. It seems preferable to select the indicator with the best properties, namely the one that captures the incidence, the intensity, and the citation inequality when $\beta=2$. Then, expression (11) can be tested using a non-parametric statistic of the degree of correspondence between two rankings (such as Kendall's tau or Spearman's coefficient), as well as a linear correlation coefficient such as Pearson's. The results are mixed. In the highimpact case, it is found that the ratio $\mu\left(x^{k}\right) / \mu(x)$ constitutes an acceptable ordinal indicator across fields only in the U.S. case, where the Kendall, Spearman, and Pearson coefficients are $0.43,0.60$, and 0.44 (all of them statistically significant), and less so in the EU, where

\footnotetext{
${ }^{8}$ To learn about the ranking of areas in every field with the information in Table 2, note that for any two areas $k$ and $l$ within the same field we have that $\mu\left(x^{k}\right) / \mu(x)>\mu\left(x^{l}\right) / \mu(x)$ implies that $\mu\left(x^{k}\right)>\mu\left(x^{l}\right)$.
} 
these coefficients are $0.24,0.32$, and 0.12 (although all of them are significant). For the RW these coefficients are tiny and statistically insignificant (p-values above 0.85). In the lowimpact case these coefficients are far from being statistically significant (only for the RW a marginally significant negative relationship can be found in a couple of cases).

From a cardinal point of view, we will assess the high-impact level achieved by different geographical areas in any field according to the two approaches by comparing the ratios $\mu\left(x^{k}\right) / \mu(x)$ and $H_{\beta}\left(x^{k} ; z\right) / H_{\beta}(x ; z)$ when $\beta=0,1$, and 2 , and the critical citation line is equal to the $80^{\text {th }}$ percentile of the world distribution. The comparison between the two approaches is illustrated in Figure 1: in each field and each geographical area, the three vertical bars reflect the ratios $H_{\beta}\left(x^{k} ; z=80^{\text {th }}\right) / H_{\beta}\left(x ; z=80^{\text {th }}\right)$ for $\beta=0,1$, and 2 ; the red color is for the U.S., blue for the EU, and green for the RW; finally, the ratios $\mu\left(x^{k}\right) / \mu(x)$ appear as a horizontal black line for each of the three geographical areas.

\section{Figure 1 around here}

In this scenario, Figure 1 clearly illustrates that judging the U.S. relative situation in terms of the MCR would seriously alter the results obtained using the high-impact indicators, especially in the case of $H_{2}$. For concreteness, columns 1 to 3 in Table 3 present the numerical differences between $H_{2}\left(x^{k} ; z=80^{t b}\right) / H_{2}\left(x ; z=80^{t b}\right)$ and $\mu\left(x^{k}\right) / \mu(x)$. The discrepancies in the U.S. (column 1) amount to a percentage between $8 \%$ and $20 \%$ in ten fields, between $20 \%$ and $30 \%$ in nine fields, and between $30 \%$ and $40 \%$ in the remaining three fields. The difference is of a smaller order of magnitude for four fields in the EU and two fields -Immunology and Computer Science- in the RW. However, the relative situation of the EU (column 2) would be more favorable according to the MCR in 11 fields by a relatively small percentage below $20 \%$, and by a rather large margin above $22 \%$ in 
seven fields. Finally, the ratio $\mu\left(x^{k}\right) / \mu(x)$ would present the RW (column 3 ) in a much more favorable position than the ratio $H_{2}\left(x^{k} ; z=80^{t h}\right) / H_{2}\left(x ; z=80^{t h}\right)$ in as many as 20 fields.

\section{Table 3 around here}

By way of example, the consequences of these differences in the measurement of the gap between the U.S. and the EU in every field are explored. Columns 1 and 2 in Table 4 present the ratios $H_{2}\left(x^{k} ; z=80^{\text {th }}\right) / H_{2}\left(x^{l} ; z=80^{\text {th }}\right)$ and $\mu\left(x^{k}\right) / \mu\left(x^{l}\right)$ for $k=$ U.S. , $l=\mathrm{EU}$, while column 3 presents the difference between these two columns in $\%$. The fact that all entries in columns 1 and 2 are greater than one indicates that both measures agree that the U.S. has a greater high-impact than the EU. It is observed that the U.S./EU gap according to our high-impact indicator $H_{2}$ is larger in every field, and in 14 out of 22 fields the difference is greater than $30 \%$.

\section{Table 4 around here}

From a cardinal point of view, the conclusion is that using only the MCR generates a very different solution from our approach to the evaluation problem. In the next Subsection, we investigate the consequences of using the remainder of the Leiden indicators.

\section{3. A Comparison of FGT Indicators and Other Leiden Indicators}

It remains to explore the possibility of completing the MCR, as is done in the Leiden triad, with the percentage of articles in the top $5 \%$ of the world distribution, $H\left(x^{k} ; z=95^{t b}\right)$, and the percentage of articles with no citations at all, $L\left(x^{k} ; z=0\right)$, defined in equations 1 and 2, respectively. We begin with a discussion of high-impact indicators. Figure 2 illustrates the comparison between the ratios $H\left(x^{k} ; z=95^{t b}\right) / H\left(x ; z=95^{t h}\right)$ and $H_{2}\left(x^{k}\right.$; $z) / H_{2}(x ; z)$ when $z$ is fixed at the $80^{\text {th }}$ and the $95^{\text {th }}$ percentile of the world citation distribution in each field. The ratios $H_{2}\left(x^{k} ; z\right) / H_{2}(x ; z)$ for the two CCLs are depicted as the 
left- and the right-handed bars in each area, while the ratio $H\left(x^{k} ; z=95^{t h}\right) / H\left(x ; z=95^{t h}\right)$ appears as the horizontal black line in each case. Note that, as indicated in Albarrán et al. (2009b), with some exceptions the impact of this change on the relative positions of the three geographical areas is relatively small. ${ }^{9}$

\section{Figure 2 around here}

As with the MCR, two ordinal comparisons are considered. Firstly, it is observed in Figure 2 that $H\left(x^{k} ; z=95^{t h}\right)$ orders all areas as $H_{2}\left(x^{k} ; z\right)$ in every field. Therefore, at least when there are only three areas in contention, the percentage of articles in the top $5 \%$ is a perfect ordinal indicator of high-impact performance in all fields. Secondly, for every area there are some similarities between the rankings of fields according to $H\left(x^{k} ; z=95^{t h}\right)$ and $H_{\beta}\left(x^{k} ; z\right)$. In particular, using $H_{2}\left(x^{k} ; z\right)$ with $z=95^{\text {th }}$ percentile, a moderately positive rank correlation for the three areas is found. Kendall's tau coefficients are $0.36,0.30$ and 0.33 , while Spearman's coefficients are $0.46,0.44$ and 0.43 (all of them statistically significant) for the US, the EU and the RW, respectively. However, all the Pearson linear correlation coefficients are insignificant.

From a cardinal point of view, one may compare $H\left(x^{k} ; z=95^{t h}\right) / H\left(x ; z=95^{t h}\right)$ with $H_{\beta}\left(x^{k} ; z k\right) / H_{\beta}(x ; z k)$ for all $k$ in any field. Since $H\left(x^{k} ; z=95^{t h}\right)$ coincides with $H o\left(x^{k} ; z=95^{t b}\right)$, and the impact of changing the critical citation line from the $95^{\text {th }}$ to the $80^{\text {th }}$ percentile is small, we proceed to compare $H\left(x^{k} ; z=95^{t h}\right) / H\left(x ; z=95^{t h}\right)$ only with $H_{2}\left(x^{k} ; z=95^{t h}\right) / H_{2}(x$; $\left.z=95^{t h}\right)$. Any discrepancy between the two measures can be unambiguously attributed to the fact that the first one does not take into account the intensity and the inequality aspects

\footnotetext{
${ }^{9}$ For example, the relative situation of the U.S. improves in 17 cases as the CCL is raised. However, it turns out that only in nine fields do these increases represent more than $15 \%$ of the level that the U.S. already achieves when the CCL is fixed at the $80^{\text {th }}$ percentile.
} 
of the high-impact phenomenon. Figure 2 illustrates that, as with MCRs, in a majority of cases judging the U.S. relative situation in terms of the $H\left(x^{k} ; z=95^{t h}\right) / H\left(x ; z=95^{t h}\right)$ ratio would underestimate the results obtained using the high-impact indicator $H_{2}\left(x^{k} ; z\right) / H_{2}(x ; z)$. However, the order of magnitude of this phenomenon is rather moderate. To see this, columns 4 to 6 in Table 3 present the differences between the two indicators in every geographical area and in every field. The worsening of the U.S. situation is below $10 \%$ in seven fields, and above this percentage in only 12 cases (column 4). The relative position of the EU (column 5) would be improved upon in fewer fields than before, and by a smaller margin (in 11 cases below 20\%, and in seven above that percentage). In the RW, the relative situation of Immunology and Computer Science is still better according to $H_{2}$, about the same in Space Science, and it is better according to the ratio $H\left(x^{k} ; z=95^{t h}\right) / H\left(x ; z=95^{t h}\right)$ in the remaining 19 fields, but by a smaller margin than in column 3 (column 6). As before, Table 4 explores the consequences of these differences for the U.S./EU gap. Columns 4 and 5 present the ratios $H_{2}\left(x^{k} ; z=95^{t h}\right) / H_{2}\left(x^{l} ; z=95^{t h}\right)$ and $H\left(x^{k} ; z=95^{t h}\right) / H\left(x^{l} ; z=95^{t h}\right)$ for $k=$ U.S. and $l=E U$, while column 6 presents the difference between these two columns in $\%$. Both measures agree that the U.S. has a greater high-impact than the EU and -except in four fields, where column 6 is negative- the gap is larger according to our high-impact indicator $H_{2}$. This difference is greater than $30 \%$ in only six cases.

As far as the low-impact phenomenon is concerned, Figure 3 illustrates the comparison between the percentage of uncited articles in each geographical area, $L\left(x^{k} ; z=0\right) / L(x ; z=$ $0)$, and the ratio $L_{2}\left(x^{k} ; z\right) / L_{2}(x ; z)$ when the critical citation line in each field is fixed at the $80^{\text {th }}$ and the $95^{\text {th }}$ percentile of the world citation distribution. The ratios $L_{2}\left(x^{k} ; z\right) / L_{2}(x ; z)$ are depicted as the colored bars in each area, while the ratio $L\left(x^{k} ; z=0\right) / L(x ; z=0)$ appears as 
the horizontal black line in each case. Note that the change in the CCL has a minimal impact on the relative positions of all areas in every field: only the RW tends to improve somewhat, while the U.S. tends to slightly worsen as the CCL is raised. In any case, the ranking of areas in terms of their contribution to world low-impact levels places the U.S. in the first place, then the EU, and finally the RW in 17 cases; in three fields there is a draw between the U.S. and the EU in first place (Mathematics, Plant and Animal Science, and Geosciences), while in two cases the UE is slightly ahead of the U.S. (Engineering, and Environmental and Ecology). Interestingly enough, this is exactly the ranking obtained with $p o\left(x^{k}\right)$. Thus, we can say that the percentage of articles without citations is an excellent ordinal indicator of low-income performance in all fields.

\section{Figure 3 around here}

For ordinal comparisons across fields, the correlation between the ratio $L\left(x^{k} ; z=\right.$ $0) / L(x ; z=0)$ and $L 2\left(x^{k} ; z\right)$ when $\beta=2$ and $z=80^{\text {th }}$ percentile indicates that this ratio is a reasonable good indicator of low-impact performance across fields in the U.S. and EU cases: the Kendall's, Spearman's and Pearson's coefficients are 0.63, 0.43 and 0.76 in the U.S., and $0.39,0.59$ and 0.49 in the EU. For the RW, the linear correlation is insignificant, and he Kendall's and Spearman's rank correlations are surprisingly negative $(-0.31$ and -0.41 with p-values around 0.05).

Finally, from a cardinal point of view the low-impact situations of areas $k$ and $l$ in any field can be compared by means of the ratios $L\left(x^{k} ; z=0\right) / L\left(x^{l} ; z=0\right)$ and $L 2\left(x^{k} ; z=\right.$ $\left.80^{t h}\right) / L_{2}\left(x^{l} ; z=80^{t h}\right)$. The more remarkable fact is that according to the percentage of articles without citations the RW is worse off than according to the low-impact indicator. The EU and, above all, the U.S. are correspondingly better off according to the $L\left(x^{k} ; z=\right.$ 
0) $/ L\left(x^{l} ; z=0\right)$ ratio. The differences in every area between $L_{2}\left(x^{k} ; z=80^{t b}\right) / L_{2}\left(x ; z=80^{t b}\right)$, and $L\left(x^{k} ; z=0\right) / L(x ; z=0)$ are in columns 7 to 9 in Table 3. They are greater than $20 \%$, approximately, in six fields for the RW (column 9), four fields for the U.S. (column 7), and two fields for the EU (column 8). The implications of such differences for the U.S./EU gap can be seen in Table 4. Columns 7 and 8 present the ratios $L_{2}\left(x^{k} ; z=80^{t b}\right) / L_{2}\left(x^{l} ; z=80^{t b}\right)$ and $L\left(x^{k} ; z=0\right) / L\left(x^{l} ; z=0\right)$ for $k=$ U.S. , $l=\mathrm{EU}$ and $z=80^{\text {th }}$ percentile, while column 9 presents the difference between these two columns in \%. Except in Engineering, the fact that all entries in columns 7 and 8 are less than one indicates that the U.S. has a lower lowimpact level than the EU. Using the percentage of articles without citations enhances the relative situation of the U.S. in 16 out of 22 cases, but except in two fields (Neurosciences and Behavioral Sciences, and Immunology) the differences between the two approaches are less than $25 \%$.

In brief, in this particular dataset the percentage of articles in the top 5\% worsens the relative situation of the U.S. and boosts that of the RW and, on most occasions, that of the $\mathrm{EU}$, while the percentage of articles without citations worsens the relative situation of the RW and improves that of the U.S. In many fields, the two approaches drastically alter the relative situation of different areas.

\section{CONCLUSIONS AND EXTENSIONS}

\section{IV.1. Conclusions}

Albarrán et al. (2011a) introduced a novel methodology for the evaluation of citation distributions in terms of a pair of high- and low-impact indicators. Albarrán et al. (2011b) applied this approach to a situation in which the world is partitioned into the U.S., the 
EU, and the RW using a large sample that covered the 22 broad scientific fields distinguished by Thomson Scientific. This paper has compared some of the main results in the latter with those obtained with alternative methodologies.

We have first examined how far we can go using only the MCR. It turns out that, when the issue is ranking only three geographical areas in each field, except in two cases whenever one area has a greater MCR than another the first has a greater high-impact and a lower low-impact than the second according to the FGT indicators. However, the ranking of fields within a given geographical area according to a normalized MCR coincides with the ranking provided by the new indicators only for high-impact levels in the U.S. For cardinal comparisons, we have confronted the MCR with high-impact indicators. For a reasonable critical citation line fixed in every field at the $80^{\text {th }}$ percentile of the world distribution, the differences between the results obtained with the two approaches are of a large order of magnitude: the discrepancies are greater than $20 \%$ half of the time. Consequently, in most fields the view according to both procedures is very different indeed. In particular, under the MCR criterion the U.S. situation systematically worsens, especially in Physics, Mathematics, and Materials Science, while the situation in the EU considerably improves in Economics and Business, and Computer Science. Correspondingly, the U.S./EU gap is greater according to our high-impact indicator in all fields, and by more than $38 \%$ in these five fields.

These results are somewhat at variance with the impression offered in Moed et al. (1995) for relatively small research units within a field: "Preliminary results suggest that the mean of the distribution correlates rather well to other statistics of the distribution, such as the median, the percentage of papers not cited, and the $90^{\text {th }}$ percentile." These important differences in the results are the consequence of the differences in the two approaches characteristics. 
In the first place, the MCR is defined over the entire distribution while the high-impact indicators are defined only over high-impact articles. In the second place, contrary to the third member of the FGT family of high-impact indicators when $\beta=2$, the MCR is not sensitive to distributional considerations. In our opinion, one plausible interpretation of the situation is that a mean-based indicator alone does not suffice to adequately represent what takes place in highly skewed citation distributions. This conclusion justifies the use of other, complementary indicators, such as those included in the Leiden triad.

The percentage of articles among the top 5\% ranks the three areas in every field exactly as the new high-impact indicators do. Similarly, the percentage of articles without citations orders all areas in every field exactly as our low-impact indicators do. At least for the U.S. and the EU, the ranking of fields according to these two percentages coincides with the ranking provided by the new high- and low-impact indicators, respectively. However, due to the fact that these percentages do not capture the intensity and the citation inequality of the phenomena in question, there are still large differences between the geographical areas' relative situation according to both approaches. Firstly, as with the MCR, the relative situation of the U.S. appears as much weaker when the percentage of articles in the top $5 \%$ is used. Correspondingly, the relative situation of the EU and, above all, the RW appears reinforced. In almost one third of the cases the differences between the two approaches are greater than $20 \%$, and there are distortions in about 13 of the 22 fields. The differences between the two approaches in the measurement of the U.S./EU gap are greater than $30 \%$ in six cases, and between $20 \%$ and $30 \%$ in 11 additional fields. Secondly, differences between the results obtained with the percentage of uncited articles or our preferred low-impact indicator are not that large: they are greater than $20 \%$ in only $16.5 \%$ of the cases, and there are serious distortions in only six fields. The main impact of using 
the Leiden rather than the low-impact indicator is to exaggerate the bad situation of the RW. Similarly, the differences between the two approaches in the measurement of the U.S./EU gap are greater than $15 \%$ in only six fields.

In brief, for the ranking of only three areas in each field, or the ranking of fields within relatively homogeneous areas such as the U.S. or the EU, following the Leiden or the new approach produces very similar results. However, considerable differences arise when the aim is the cardinal comparison of each area's relative situation. As Moed et al. (1995) eloquently point out (after evaluating research groups for many years using the MCR and the percentage of articles without citations), "An important step would in fact be to develop indicators of the impact of a group's very best articles, and compare the results to those obtained by applying the citation per publication ratio." This is indeed the step taken in important papers that have been already referred to, namely, Tijssen et al. (2002) and van Leeuwen et al. (2003). In our view, this is also the step that our methodology has attempted to take by introducing high-impact indicators. The difference, of course, is that we have provided an integrated framework in which the entire citation distribution can be conveniently described by a pair of high- and low-impact indices whose properties have been fully described and have proved to be useful in the empirical work. Furthermore, as indicated in Albarrán et al (2011b), the approach can be profitably extended to cover the analysis of inter-temporal trends, as well as the search for dominance results valid for any critical citation line and any high- or low-impact indicator in a wide class of admissible indices.

\section{2. Extensions}

1. It should be clear that one the novelties of the approach advocated here is that members of the FGT families when the parameter $\beta$ is equal or greater than two vary 
directly with citation inequality. However, the sensitivity to citation inequality may result in drawbacks of its own. FGT and similar high-impact indices may become very sensitive to extreme observations, namely, to the presence of one or a few articles with a phenomenal number of citations (see Albarrán et al., 2011b). More research is needed to learn about the robustness of the results obtained with FGT indicators to the trimming of a handful of observations. At the same time, it might be interesting to use FGT highimpact measures in connection with other indicators that are robust to extreme observations.

2. In the first application of the new approach, it has been natural to exploit the additive decomposability of the FGT indicators to a small partition of the world into three geographical areas. Beyond the extension to the many countries case, the practical interest of the new approach should also be tested in the evaluation of smaller research units, such as the research groups or institutes that have occupied much of the attention of the Leiden group and other authors in bibliometrics.

3. Even more necessary is to address the comment from one referee that the main results of the paper could be translated as that the U.S. is disproportionally active in the highest impact subject categories within the 22 broad fields selected. Further research should start by identifying homogeneous fields with Web of Science categories or similar sub-field notions in other datasets, and should also confront the aggregation problems and alternatives from the sub-field to the broad field level in the presence of the multiple assignment of articles to sub-fields at the lowest aggregation level. 


\section{REFERENCES}

Aksnes, D., and G. Sivertsen (2004), “The Effect of Highly Cited Papers on National Citation Indicators", Scientometrics, 59: 213-224.

Albarrán, P. and J. Ruiz-Castillo (2009), "References Made and Citations Received By Scientific Articles", forthcoming in Journal of the American Society for Information Science and Technology (DOI: 10.1002/asi.21448).

Albarrán, P., J. Crespo, I. Ortuño, and J. Ruiz-Castillo (2010a), “The Skewness of Science In 219 Sub-fields and a Number of Aggregates”, Working Paper 10-38, Universidad Carlos III.

Albarrán, P., J. Crespo, I. Ortuño, and J. Ruiz-Castillo (2010b), “A Comparison of the Scientific Performance of the U.S. and the European Union at the Turn of the 21st Century”, Scientometrics, 85: 329-344.

Albarrán, P., I. Ortuño, and J. Ruiz-Castillo (2010c), “Average-based versus High- and Low-impact Indicators for the Evaluation of Scientific Distributions”, Working Paper 10-40, Universidad Carlos III.

Albarrán, P., I. Ortuño, and J. Ruiz-Castillo (2011a), “The Measurement of Low- and High-impact in Citation Distributions: Technical Results”, Working Paper 09-57, Economics Series 35, Universidad Carlos III, Journal of Informetrics, 5: 48-63.

Albarrán, P., I. Ortuño, and J. Ruiz-Castillo (2009b), "High- and Low-impact Citation Measures: Empirical Applications”, Working Paper 09-58, Economics Series 36, Universidad Carlos III, Journal of Informetrics, 5: 122-145.

Bornmann, L., R. Mutz, C. Neuhaus, and H-D. Daniel (2008), "Citation Counts for Research Evaluation: Standards of Good Practice for Analyzing Bibliometric Data and Presenting and Interpreting Results”, Etbics in Science and Environmental Politics, 18: 93-102.

Foster, J.E., J. Greeer, and E. Thorbecke (1984), “A Class of Decomposable Poverty Measures”, Econometrica, 52: 761-766.

Glänzel, W. (2002), "Coauthorship Patterns and Trends in the Sciences (1980-1998): A Bibliometric Study with Implications for Database Indexing and Search Strategies”, Library Trends, 50: 461-473.

Glänzel, W. (2007), "Characteristic Scores and Scales. A Bibliometric Analysis of Subject Characteristics Based on Long-term Citation Observation”, Journal of Informetrics, 1: 92-102.

Glänzel, W., A. Schubert, and T. Braun (2002), “A Relational Charting Approach to the World of Basic Research In Twelve Science Fields at the End of the Second Millennium”, Scientometrics, 55: 335-348.

Moed, H. F., W. J. Burger, J. G. Frankfort, and A. F. J. van Raan (1985), “The Use of Bibliometric Data for the Measurement of University Research Performance”, Research Policy, 14: 131-149.

Moed, H. F., and A. F. J. van Raan (1988), "Indicators of Research Performance", in A. F. J. van Raan (ed), Handbook of Quantitative Studies of Science and Technology, North Holland: 177-192.

Moed, H. F., R. E. De Bruin, and T. van Leeuwen (1995), "New Bibliometrics Tools for the Assessment of National Research Performance: Database Description, Overview of Indicators, and First Applications", Scientometrics, 133: 381-422.

Schubert, A., and T. Braun (1986), "Relative Indicators and Relational Charts for Comparative Assessment of Publication Output and Citation Impact”, Scientometrics, 9: 281-291. 
Schubert, A., W. Glänzel and T. Braun (1987), “A New Methodology for Ranking Scientific Institutions”, Scientometrics, 12: 267-292.

Seglen, P. (1992), "The Skewness of Science", Journal of the American Society for Information Science, 43: 628-638.

Tijssen, R, M. Visser, and T. van Leeuwen (2002), "Benchmarking International Scientific Excellence: Are Highly Cited Research Papers an Appropriate Frame of Reference”, Scientometrics, 54: 381-397.

Van Leeuwen, T., M. Visser, H. Moed, T. Nederhof, and A, van Raan (2003), "The Holy Grail of Science Policy: Exploring and Combining Bibliometric Tools In Search of Scientific Excellence", Scientometrics, 57: 257-280.

Van Raan, A. F. J. (2004), "Measuring Science", in H. F. Moed et al. (eds.), Handbook of Quantitative Science and Technology Research: 19-50. 
Table 1A. The Ratio Of Observed Contributions (OC) To High-impact Overall Levels To Expected Contributions (EC) By Geographical Area In Every Scientific Field*

The OC Captures Only the Incidence $(\beta=0)$, the Incidence and the Intensity $(\beta=1)$, or the Incidence, the Intensity, and the Inequality $(\beta=2)$ Of the High-impact Phenomenon

\section{UNITED STATES EUROPEAN UNION REST OF THE WORLD \\ $\beta=0 \quad \beta=1 \quad \beta=2 \quad \beta=0 \quad \beta=1 \quad \beta=2 \quad \beta=0 \beta=1 \quad \beta=2$}

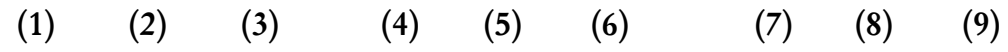

LIFE SCIENCES

$\begin{array}{llll}1.30 & 1.42 & 1.55 & 0.95\end{array}$

$\begin{array}{lll}1.45 & 1.56 \quad 1.73\end{array}$

$\begin{array}{lll}0.75 & 0.69 & 0.66\end{array}$

(2) Biology \& Biochemistry

$\begin{array}{lll}1.32 & 1.45 \quad 1.58\end{array}$

0.95

0.94

$0.90 \quad 0.86$

$0.65 \quad 0.60 \quad 0.49$

(3) Neuroscience \& Behav. Sc.

1.30

$1.39 \quad 1.48$

0.95

$0.87 \quad 0.78$

$\begin{array}{lll}0.68 & 0.61 & 0.54\end{array}$

$\begin{array}{llll}\text { (5) Psychiatry \& Psychology } & 1.12 & 1.20 & 1.31\end{array}$

0.95

$0.89 \quad 0.81$

$\begin{array}{lll}0.67 & 0.63 & 0.60\end{array}$

(6) Pharmacology \& Toxicology

$1.42 \quad 1.55 \quad 1.57$

1.05

$0.88 \quad 0.73$

$\begin{array}{lll}0.79 & 0.70 & 0.64\end{array}$

(7) Microbiology

$\begin{array}{lll}1.43 & 1.58 & 1.75\end{array}$

1.01

$1.04 \quad 1.11$

$\begin{array}{lll}0.69 & 0.61 & 0.55\end{array}$

(8) Immunology

1.26

$1.32 \quad 1.34$

0.92

$0.96 \quad 0.88$

$\begin{array}{lll}0.63 & 0.56 & 0.51\end{array}$

PHYSICAL SCIENCES

(9) Chemistry

(10) Physics

(11) Computer Science

$\begin{array}{lll}1.60 & 1.96 & 1.99\end{array}$

$\begin{array}{lll}1.13 & 1.04 & 1.16\end{array}$

$0.76 \quad 0.76$

0.88

(12) Mathematics

(13) Space Science

OTHER NATURAL SCIENCES

(14) Engineering

(15) Plant \& Animal Science

(16) Materials Science

$\begin{array}{lll}1.46 & 1.71 \quad 2.08\end{array}$

$\begin{array}{lll}1.13 & 1.09 & 0.96\end{array}$

$\begin{array}{lll}0.70 & 0.61 & 0.53\end{array}$

$\begin{array}{lll}1.31 & 1.53 & 1.61\end{array}$

$\begin{array}{lll}0.95 & 0.87 & 0.53\end{array}$

$\begin{array}{lll}0.72 & 0.64 & 0.57\end{array}$

$\begin{array}{lll}1.25 & 1.47 \quad 1.97\end{array}$

$\begin{array}{lll}1.10 & 1.04 & 0.81\end{array}$

$\begin{array}{lll}0.78 & 0.66 & 0.92\end{array}$

$\begin{array}{lll}1.34 & 1.39 & 1.39\end{array}$

0.97

$0.96 \quad 0.98$

$\begin{array}{lll}0.76 & 0.66 & 0.54\end{array}$

$\begin{array}{lll}0.68 & 0.65 & 0.63\end{array}$

(17) Geoscience

(18) Environment \& Ecology

$\begin{array}{lll}1.21 & 1.37 & 1.62\end{array}$

$\begin{array}{lll}1.11 & 1.09 & 1.04\end{array}$

$\begin{array}{lll}0.81 & 0.72 & 0.61\end{array}$

$\begin{array}{lll}1.21 & 1.33 & 1.57\end{array}$

$\begin{array}{lll}1.18 & 1.19 \quad 1.07\end{array}$

$0.75 \quad 0.67$

0.60

$\begin{array}{lll}1.37 & 1.74 & 2.27\end{array}$

$\begin{array}{lll}1.12 & 1.05 & 0.94\end{array}$

$0.82 \quad 0.73$

0.62

$\begin{array}{lll}1.37 & 1.49 & 1.55\end{array}$

$\begin{array}{lll}1.06 & 1.00 & 0.98\end{array}$

$\begin{array}{lll}0.69 & 0.65 & 0.63\end{array}$

$\begin{array}{lll}1.21 & 1.32 & 1.52\end{array}$

$\begin{array}{lll}1.06 & 1.01 & 0.94\end{array}$

$\begin{array}{lll}0.77 & 0.71 & 0.60\end{array}$

$\begin{array}{lll}1.27 & 1.47 & 1.67\end{array}$

$\begin{array}{lll}1.21 & 1.18 \quad 1.17\end{array}$

$\begin{array}{lll}1.32 & 1.32 & 1.12\end{array}$

$0.70 \quad 0.62$

0.52

1.89

2.25

2.52

$\begin{array}{lll}1.13 & 1.22 & 1.33\end{array}$

$\begin{array}{lll}0.95 & 0.86 & 0.74\end{array}$

$0.53 \quad 0.38$

0.33

IAL SCIENCES

(21) Social Sciences, General

(22) Economics \& Business

$\begin{array}{lll}0.81 & 0.69 & 0.62\end{array}$

$\begin{array}{lll}0.75 & 0.62 & 0.49\end{array}$

$0.64 \quad 0.53 \quad 0.46$

* In any field and any column, a cell value is greater than, equal to, or smaller than one when the geographical area's observed contribution (OC) to the world high-impact level is greater than, equal to, or smaller than the area's publication share (or expected contribution, EC) in the extended citation distribution.

In row (1) Clinical Medicine, for example, when only the incidence of the high-impact phenomenon is taken into account: (i) the U.S. OC according to $H_{0}$ is $30 \%$ above the corresponding EC; (ii) the EU OC according to $H_{0}$ is $5 \%$ below the corresponding EC, and (iii) the RW OC according to that index is $25 \%$ below the corresponding EC. When the aggregate gap between the citations received by high-impact articles and the CCL is taken into account, the U.S. OC according to $H_{1}$ is $42 \%$ above the EC. Finally, when the citation inequality among high-impact articles is also taken into account, the U.S. OC according to $H_{2}$ is $55 \%$ above its EC. 
Table 1B. The Ratio Of Observed Contributions (OC) To Low-impact Overall Levels To Expected Contributions (EC) By Geographical Area In Every Scientific Field*

The OC Captures Only the Incidence $(\beta=0)$, the Incidence and the Intensity $(\beta=1)$, or the Incidence, the Intensity, and the Inequality $(\beta=2)$ Of the Low-impact Phenomenon

\section{UNITED STATES EUROPEAN UNION \\ $\beta=0 \quad \beta=1 \quad \beta=2 \quad \beta=0 \quad \beta=1 \quad \beta=$ \\ $\beta=0 \quad \beta=1 \quad \beta=2$}

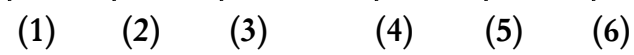

LIFE SCIENCES

(1) Clinical Medicine

$0.87 \quad 0.84$

.04

(2) Biology \& Biochemistry

(3) Neuroscience \& Behav. Sc.

(4) Molecular Biology \& Genetics

(5) Psychiatry \& Psychology

(6) Pharmacology \& Toxicology

(7) Microbiology

(8) Immunology

(7)

(8)

(9)

\section{PHYSICAL SCIENCES}

(9) Chemistry

(10) Physics

(11) Computer Science

(12) Mathematics

(13) Space Science

OTHER NATURAL SCIENCES

(14) Engineering $\quad 0.96$

(15) Plant \& Animal Science

(16) Materials Science

(17) Geoscience

(18) Environment \& Ecology

(19) Agricultural Sciences

(20) Multidisciplinary

SOCIAL SCIENCES

(21) Social Sciences, General

(22) Economics \& Business
0.92

0.89

$\begin{array}{ll}1.01 & 1.03\end{array}$

1.04

0.92

0.92

0.97

0.90

0.89

0.93

0.80

$0.86 \quad 0.82$

1.01

1.01

0.99

0.98

$0.85 \quad 0.82$

1.01

1.01

1.00

0.99

$\begin{array}{ll}0.94 & 0.93 \\ 0.86 & 0.84\end{array}$

0.99

1.00

1.02

1.03

0.97

0.96

1.03

1.04

0.96

0.94

1.04

$\begin{array}{ll}1.07 & 1.11\end{array}$

1.13

$\begin{array}{lll}1.08 & 1.18 & 1.24\end{array}$

$\begin{array}{lll}1.08 & 1.16 & 1.20\end{array}$

$\begin{array}{lll}1.09 & 1.19 & 1.24\end{array}$

$\begin{array}{lll}1.05 & 1.10 & 1.12\end{array}$

$\begin{array}{lll}1.07 & 1.11 & 1.13\end{array}$

$\begin{array}{lll}1.10 & 1.21 & 1.27\end{array}$

$\begin{array}{lll}1.06 & 1.13 & 1.16\end{array}$

0.85

$\begin{array}{ll}0.76 & 0.72\end{array}$

$\begin{array}{lll}0.97 & 0.90 & 0.85\end{array}$

$\begin{array}{lll}1.08 & 1.15 & 1.19\end{array}$

0.89

$0.83 \quad 0.80$

$\begin{array}{ll}0.97 & 0.93\end{array}$

0.92

1.07

1.05

0.93

0.95

0.90

$0.90 \quad 0.89$

$\begin{array}{lll}0.98 & 0.95 & 0.93\end{array}$

$\begin{array}{lll}1.05 & 1.10 & 1.13\end{array}$

$\begin{array}{lll}1.01 & 1.01 & 1.02\end{array}$

$1.09 \quad 1.17$

1.21

$0.94 \quad 0.94$

0.98

0.94

0.93

$\begin{array}{lll}1.04 & 1.07 & 1.08\end{array}$

$\begin{array}{lll}0.96 & 0.92 & 0.90\end{array}$

$\begin{array}{lll}1.06 & 1.11 & 1.14\end{array}$

0.91

$\begin{array}{ll}0.91 & 0.89 \\ 0.86 & 0.84\end{array}$

0.97

0.94

0.92

$\begin{array}{lll}1.05 & 1.08 & 1.09\end{array}$

0.90

$\begin{array}{ll}0.82 & 0.79\end{array}$

0.98

0.93

$0.99 \quad 0.95$

0.90

$\begin{array}{lll}1.08 & 1.18 & 1.23\end{array}$

$\begin{array}{lll}1.05 & 1.11 & 1.13\end{array}$

0.95

$0.93 \quad 0.92$

0.95

0.89

0.93

$\begin{array}{lll}1.08 & 1.16 & 1.20\end{array}$

0.80

0.72

0.93

0.91

0.91

$\begin{array}{lll}1.11 & 1.14 & 1.16\end{array}$

$\begin{array}{lll}0.97 & 0.96 & 0.95\end{array}$

$\begin{array}{lll}1.01 & 1.00 & 1.00\end{array}$

$\begin{array}{lll}1.06 & 1.09 & 1.11\end{array}$

$\begin{array}{lll}0.94 & 0.90 & 0.89\end{array}$

$1.08 \quad 1.13 \quad 1.16$ $\begin{array}{lll}1.01 & 1.01 & 1.01\end{array}$

* In any field and any column, a cell value is greater than, equal to, or smaller than one when the geographical area's observed contribution (OC) to the world low-impact level is greater than, equal to, or smaller than the area's publication share (or expected contribution, EC) in the extended citation distribution.

In row (1) Clinical Medicine, for example, when only the incidence of the low-impact phenomenon is taken into account it can be seen that the U.S., the EU, and the RW OC according to $L_{0}$ is $8 \%$ below, $1 \%$ above, and $7 \%$ above the corresponding EC, respectively. 
Table 2. Contributions To World MCRs, the Top 5\% of Highly Cited Articles, and Articles Without Citations By Geographical Area*

(A) Contributions To MCRs In Area $k, M C R\left(x^{k}\right) / M C R(x), k=U . S$., $E U, R W$

(B) Contributions To the Top 5\% of Highly Cited Articles In Area $k, H\left(x^{k}, z=95^{t b}\right) / H\left(x, z=95^{t b}\right), k=U . S$., $E U, R W$

(C) Contributions To the Articles With Zero Citations In Area $k, L\left(x^{k}, z=0\right) / L\left(x^{k}, z=0\right), k=U . S ., E U, R W$

\section{LIFE SCIENCES}

Clinical Medicine

Biology \& Biochemistry

Neuroscience \& Behav. Science

Molecular Biology \& Genetics

Psychiatry \& Psychology

Pharmacology \& Toxicology

Microbiology

Immunology

PHYSICAL SCIENCES

Chemistry

Physics

Computer Science

Mathematics

Space Science

OTHER NATURAL SCIENCES
(A)

U.S. EU RW
(1)
(2)
(3)

$\begin{array}{lll}1.27 & 0.94 & 0.79 \\ 1.33 & 0.97 & 0.74 \\ 1.24 & 0.95 & 0.77 \\ 1.25 & 0.96 & 0.73 \\ 1.11 & 0.93 & 0.82 \\ 1.28 & 1.04 & 0.79 \\ 1.30 & 1.02 & 0.73 \\ 1.19 & 0.94 & 0.84\end{array}$

1.44

1.58

1.48

1.40

1.20

1.61

1.61

1.34

U.S.

(4)

(B)

(C)

U.S. EU RW

(7)

(8)

(9)

$\begin{array}{llllllllll}\text { Engineering } & 1.19 & 1.09 & 0.83 & 1.39 & 1.09 & 0.71 & 0.95 & 0.90 & 1.13 \\ \text { Plant \& Animal Science } & 1.18 & 1.13 & 0.80 & 1.32 & 1.21 & 0.66 & 0.83 & 0.85 & 1.28 \\ \text { Materials Science } & 1.41 & 1.07 & 0.83 & 1.76 & 1.06 & 0.72 & 0.80 & 0.89 & 1.16 \\ \text { Geoscience } & 1.29 & 1.05 & 0.76 & 1.54 & 0.97 & 0.64 & 0.73 & 0.75 & 1.49 \\ \text { Environment \& Ecology } & 1.15 & 1.05 & 0.83 & 1.35 & 0.99 & 0.71 & 0.92 & 0.86 & 1.26 \\ \text { Agricultural Sciences } & 1.26 & 1.15 & 0.75 & 1.45 & 1.21 & 0.60 & 0.74 & 0.78 & 1.37 \\ \text { Multidisciplinary } & 1.91 & 1.24 & 0.54 & 2.33 & 1.28 & 0.36 & 0.63 & 0.92 & 1.19 \\ \text { SOCIAL SCIENCES } & & & & & & & & & \\ \text { Social Sciences, General } & 1.11 & 0.95 & 0.78 & 1.24 & 0.84 & 0.59 & 0.94 & 1.01 & 1.17 \\ \text { Economics \& Business } & 1.25 & 0.83 & 0.70 & 1.46 & 0.66 & 0.51 & 0.86 & 1.06 & 1.26\end{array}$

* In any field and any column, a cell value is greater than, equal to, or smaller than one when the geographical area's observed contribution (OC) to the world level is greater than, equal to, or smaller than the area's publication share (or expected contribution, EC) in the extended citation distribution. 
Figure 1. Relative Contributions To World High-impact Levels By the U.S. (red), the EU (blue), and the RW (green) According To Members of the FGT Family of High-impact Indicators. Relative Mean Citation Rates Appear As Black Horizontal Lines
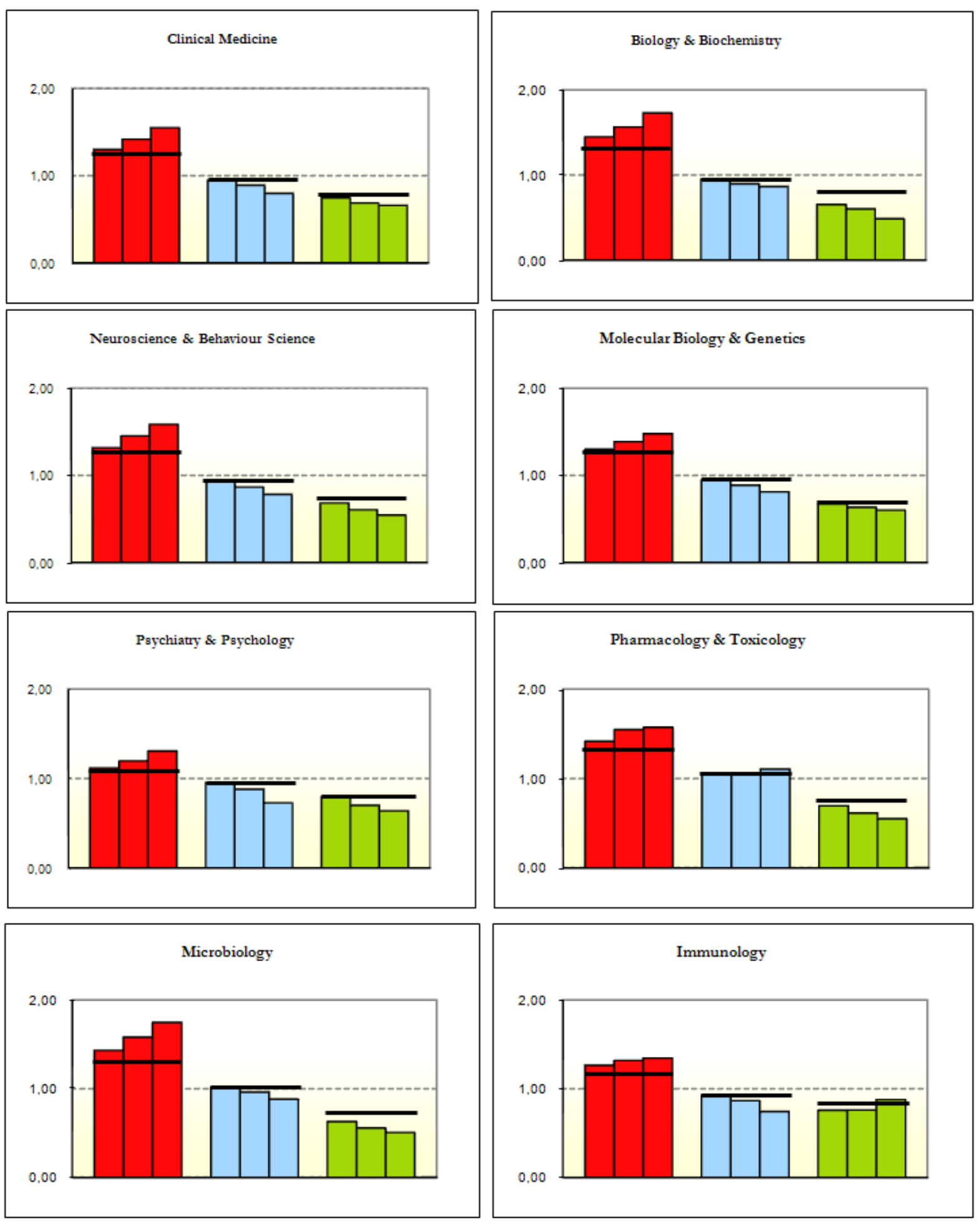
Figure 1. Relative Contributions To World High-impact Levels By the U.S. (red), the EU (blue), and the RW (green) According To Members of the FGT Family of High-impact Indicators. Relative Mean Citation Rates Appear As Black Horizontal Lines
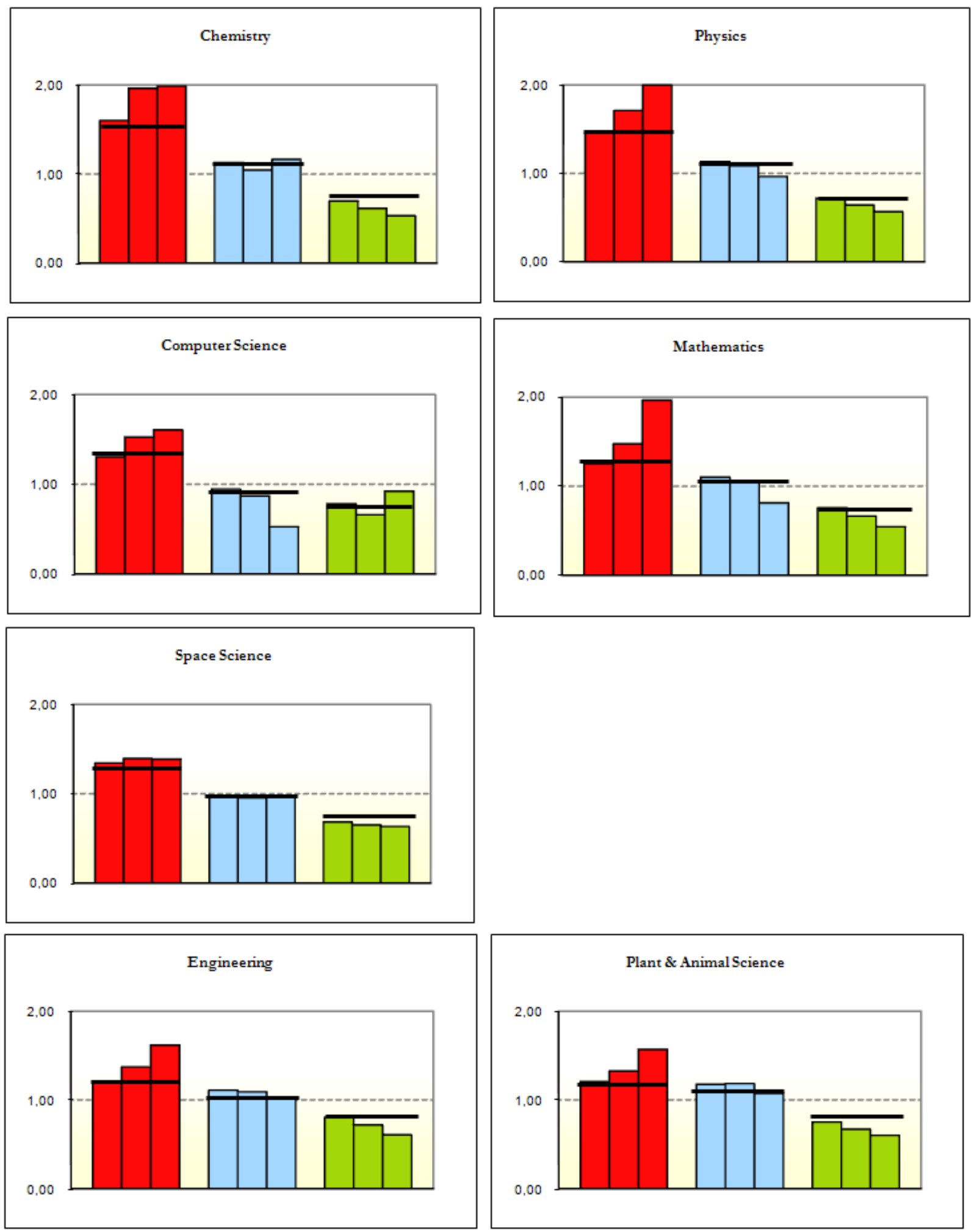
Figure 1. Relative Contributions To World High-impact Levels By the U.S. (red), the EU (blue), and the RW (green) According To Members of the FGT Family of High-impact Indicators. Relative Mean Citation Rates Appear As Black Horizontal Lines
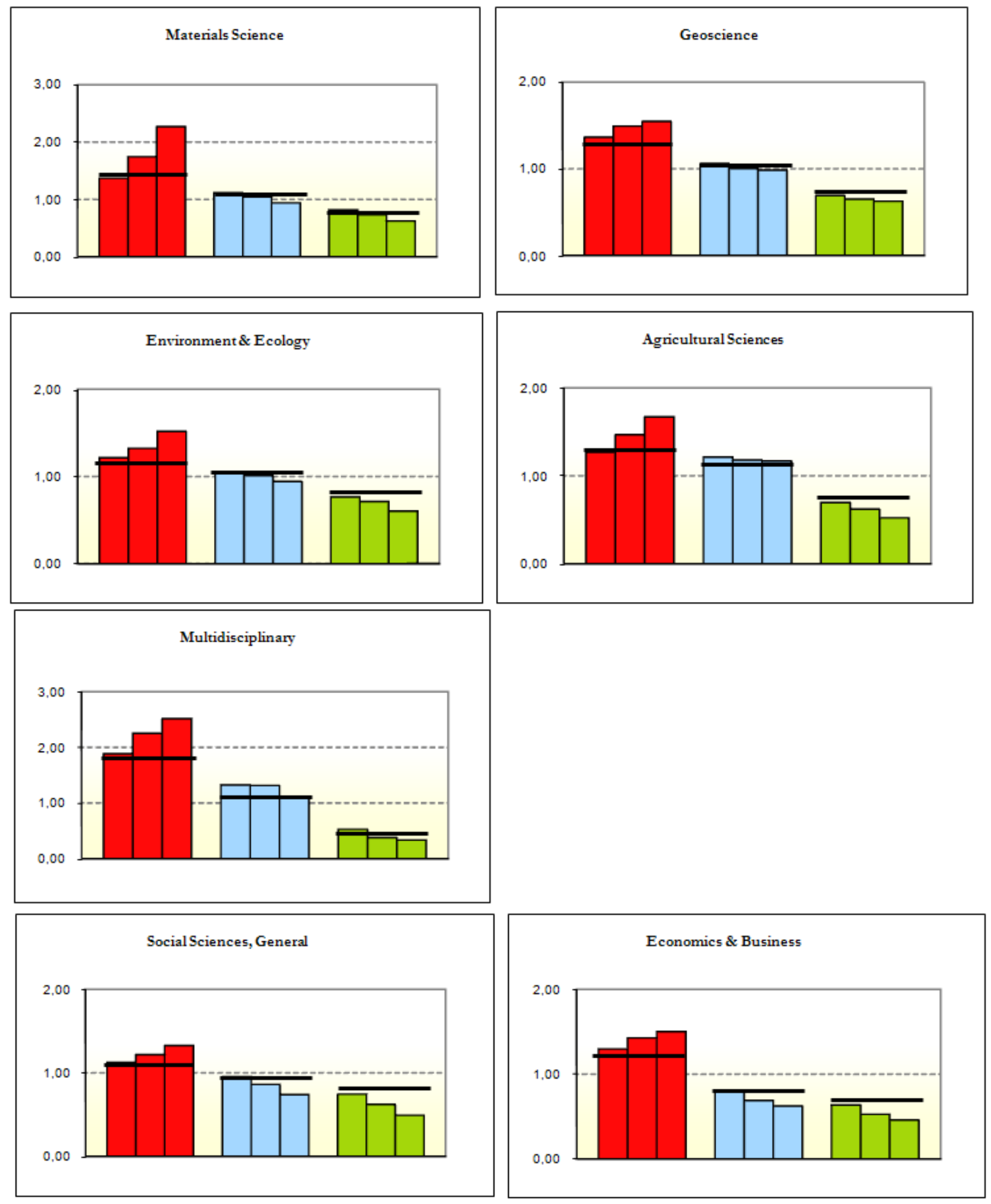
Table 3. Differences Between the Contributions To High- and Low-impact Levels In Area $k=$ U.S., EU, and RW According To the Two Approaches
(A) Difference In \% Between $H_{2}\left(x^{k} ; z=80^{\text {th }}\right) / H_{2}\left(x ; z=80^{\text {th }}\right)$ and $\mu\left(x^{k}\right) / \mu(x)$
(B) Difference In \% Between $H_{2}\left(x^{k} ; z=95^{\text {th }}\right) / H_{2}\left(x ; z 95^{\text {th }}\right)$ and $H\left(x^{k}, z=95^{\text {th }}\right) / H\left(x, z=95^{\text {th }}\right)$
(C) Difference In \% Between $L_{2}\left(x^{k} ; z=80^{\text {th }}\right) / L_{2}\left(x ; z=80^{\text {th }}\right)$ and $L\left(x^{k}, z=0\right) / L(x, z=0)$

(A)

U.S. EU RW

(1) (2) (3)

18

Clinical Medicine

Biology \& Biochemistry

Neuroscience \& Behav. Science

Molecular Biology \& Genetics

Psychiatry \& Psychology

Pharmacology \& Toxicology

Microbiology

Immunology

PHYSICAL SCIENCES

Chemistry

Physics

Computer Science

Mathematics

Space Science

OTHER NATURAL SCIENCES

Engineering

Plant \& Animal Science

Materials Science

Geoscience

Environment \& Ecology

Agricultural Sciences

Multidisciplinary

SOCIAL SCIENCES

Social Sciences, General

Economics \& Business

$23.2 \quad-12.7 \quad-51.9$

$21.8 \quad-21.4 \quad-41.4$

$15.6 \quad-18.0-22.0$

$14.7 \quad-28.3 \quad-28.0$

$18.8 \quad 6.2 \quad-44.3$

$25.5 \quad-15.4 \quad-44.5$

$\begin{array}{lll}11.7 & -26.0 \quad 4.2\end{array}$

(B)

U.S. EU RW

(4) (5) (6)

$-15.4 \quad-2.6$

$-2.4 \quad-38.8$

11.5

11.5

$-18.1$

$-13.7$

8.7

$-14.7$

$-7.7$

12.7

$-46.3$

$-8.8$

$11.5-13.6$

$-3.7$

14.2

$-18.7$

$-14.0$

1.0

$-32.8 \quad 26.0$

$$
24.2
$$

$31.2 \quad-12.8 \quad-34.3$

$-5.6$

$16.5 \quad-76.6 \quad 16.7$

22.1

$36.2 \quad-31.2 \quad-44.2$

8.0

$0.4 \quad-18.5$

$-3.6$

\section{6.}

$24.9 \quad-4.80 \quad-33.6$

20.7

37.9

$\begin{array}{lll}16.6 & -6.6 & -20.8\end{array}$

$24.2 \quad-11.0 \quad-37.9$

$\begin{array}{lll}24.3 & 1.6 & -42.9\end{array}$

$\begin{array}{lll}24.0 & -10.7 \quad-62.7\end{array}$

$\begin{array}{lll}16.0 & -28.0 & -58.7 \\ 16.7 & -34.0 & -52.8\end{array}$
4.3

33.3

$15.7-15.8$

$-20.0 \quad-14.8$

$-69.1 \quad 30.6$

$-50.9 \quad-33.1$

$\begin{array}{ll}3.9 & 0.2\end{array}$

$-9.0 \quad-29.1$

$-22.2-15.1$

$-19.0 \quad-26.6$

$1.8 \quad-3.2$

$-9.8 \quad-33.2$

$-2.9 \quad-30.5$

$-22.3-8.8$

9.8

10.6

4.2

$\begin{array}{rr}-24.8 & -40.6 \\ -7.7 & -17.7\end{array}$

U.S.

(7)

(8)

(9)
(C)

$\begin{array}{rrr}22.7 & -8.3 & -7.1 \\ 42.7 & 11.3 & -26.5 \\ 26.6 & -8.6 & -22.1 \\ 16.0 & 20.8 & -42.1 \\ 7.5 & -6.6 & -13.3 \\ -9.4 & 0.5 & 0.5 \\ 29.9 & 14.7 & -35.9 \\ 20.0 & -7.6 & -19.9\end{array}$

$17.4 \quad 19.0 \quad-16.1$

$\begin{array}{lll}7.4 & 2.5 & -10.5\end{array}$

$\begin{array}{lll}1.8 & 0.1 & -4.2\end{array}$

$\begin{array}{lll}2.8 & 3.3 \quad-6.5\end{array}$

$10.4 \quad-8.3 \quad-13.7$

$\begin{array}{rrr}-1.4 & 3.7 & -4.7 \\ 7.2 & 5.2 & -11.9 \\ 5.2 & 3.8 & -6.0 \\ 7.6 & 16.3 & -21.5 \\ -0.1 & 7.4 & -11.6 \\ 11.4 & 8.9 & -13.9 \\ 9.4 & -1.3 & -2.8\end{array}$

$\begin{array}{lll}0.7 & -0.8 & -5.0\end{array}$

$\begin{array}{lll}3.7 & 0.0 & -8.3\end{array}$


Table 4. The U.S./EU Gap According to Different Indicators

(1) High-impact Indicator: $H_{2}\left(x^{U . S .} ; z=80^{\text {th }}\right) / H_{2}\left(x^{E U} ; z=80^{\text {th }}\right)$

(2) Mean Citation Ratio: $\mu\left(x^{U . S .}\right) / \mu\left(x^{E U}\right)$

$(3)=100[(1)-(2)] /(2)$, i.e. Difference in $\%$

(4) High-impact Indicator: $H_{2}\left(x^{U . S .} ; z=95^{\text {th }}\right) / H_{2}\left(x^{E U} ; z=95^{\text {th }}\right)$

(5) Percentage In the Top 5\% of Highly Cited Articles: $H\left(x^{\text {U.S. }}, z=95^{\text {th }}\right) / H\left(x^{E U}, z=95^{\text {th }}\right)$

$(6)=100[(4)-(5)] /(5)$, i.e. Difference in \%

(7) Low-impact Indicator: $L_{2}\left(x^{\text {U.S. }} ; z=80^{\text {th }}\right) / L_{2}\left(x^{E U} ; z=80^{\text {th }}\right)$

(8) Percentage of Articles With Zero Citations: $L\left(x^{U . S .}, z=0\right) / H\left(x^{E U}, z=0\right)$

$(9)=100[(7)-(8)] /(8)$, i.e. Difference in $\%$

\section{SCIENTIFIC FIELDS \\ LIFE SCIENCES}

Clinical Medicine

Biology \& Biochemistry

Neuroscience \& Behav. Science

Molecular Biology \& Genetics

Psychiatry \& Psychology

Pharmacology \& Toxicology

Microbiology

Immunology

PHYSICAL SCIENCES

Chemistry

Physics

Computer Science

Mathematics

Space Science

OTHER PH. SCIENCES

Engineering

Plant \& Animal Science

Materials Science

Geoscience

Environment \& Ecology

Agricultural Sciences

Multidisciplinary

SOCIAL SCIENCES

Social Sciences, General

Economics \& Business
$H_{2}, z=80^{\text {th }}$

vs. MCR

(1)

(2)

(3)

$\begin{array}{lll}1.94 & 1.36 & 30.2 \%\end{array}$

$\begin{array}{lll}2.00 & 1.36 & 31.8 \%\end{array}$

$\begin{array}{lll}2.03 & 1.31 & 35.6 \%\end{array}$

$\begin{array}{lll}1.82 & 1.30 \quad 28.5 \%\end{array}$

$\begin{array}{lll}1.79 & 1.19 & 33.5 \%\end{array}$

$\begin{array}{lll}1.42 & 1.23 & 13.4 \%\end{array}$

$\begin{array}{lll}1.98 & 1.28 & 35.5 \%\end{array}$

$\begin{array}{lll}1.81 & 1.27 \quad 29.9 \%\end{array}$

$\begin{array}{lll}1.71 & 1.37 & 19.4 \%\end{array}$

$\begin{array}{lll}2.15 & 1.32 & 39.0 \%\end{array}$

$3.06 \quad 1.44 \quad 52.7 \%$

$2.42 \quad 1.18 \quad 51.4 \%$

$\begin{array}{lll}1.42 & 1.31 \quad 7.6 \%\end{array}$

$\begin{array}{lll}1.56 & 1.10 \quad 29.7 \%\end{array}$

$1.46 \quad 1.05$

$2.42 \quad 1.32$

$28.3 \%$

$45.2 \%$

$\begin{array}{ll}1.57 & 1.23\end{array}$

$21.8 \%$

$1.61 \quad 1.10$

$31.7 \%$

$1.43 \quad 1.10$

$23.1 \%$

$2.24 \quad 1.54$

$31.3 \%$

$\begin{array}{lll}1.79 & 1.18 & 34.3 \%\end{array}$

$2.41 \quad 1.50 \quad 37.9 \%$
$H_{2}, z=95^{\text {th }}$

vs. $H\left(x, z=95^{\text {th }}\right)$

(4)

(5)

(6)

(7)

(8) (9)

(8) (9)

$L_{2}, z=80^{\text {th }}$

vs. $L(x, z=0)$

$\begin{array}{lll}2.04 & 1.62 \quad 20.8 \%\end{array}$

$\begin{array}{lll}0.81 & 0.67 & 17.5 \%\end{array}$

$2.09 \quad 1.80 \quad 13.7 \%$

$0.76 \quad 0.65$

$15.4 \%$

$2.30 \quad 1.71 \quad 25.3 \%$

$0.81 \quad 0.54$

$32.7 \%$

$2.00 \quad 1.58 \quad 20.9 \%$

$0.83 \quad 0.88$

$-6.2 \%$

$\begin{array}{lll}2.26 & 1.35 & 40.2 \%\end{array}$

$0.89 \quad 0.78$

$13.2 \%$

$\begin{array}{lll}1.34 & 1.57 & -17.3 \%\end{array}$

$0.88 \quad 0.96$

$-9.5 \%$

$\begin{array}{lll}2.34 & 1.70 \quad 27.5 \%\end{array}$

$0.80 \quad 0.66$

$18.1 \%$

$2.05 \quad 1.52 \quad 25.6 \%$

$\begin{array}{ll}0.80 & 0.59\end{array}$

$25.7 \%$

$\begin{array}{lll}1.57 & 1.96 & -25.4 \%\end{array}$

$\begin{array}{ll}0.84 & 0.86\end{array}$

$-2.6 \%$

$\begin{array}{lll}2.32 & 1.52 & 34.7 \%\end{array}$

0.87

0.83

$5.2 \%$

$\begin{array}{lll}3.17 & 1.79 & 43.6 \%\end{array}$

$0.88 \quad 0.87$

$1.5 \%$

$3.11 \quad 1.38 \quad 55.7 \%$

$0.96 \quad 0.96$

$-0.6 \%$

$\begin{array}{lll}1.38 & 1.49 & -7.8 \%\end{array}$

$0.76 \quad 0.62$

$18.0 \%$

$\begin{array}{lll}1.75 & 1.27 & 27.1 \% \\ 1.73 & 1.09 & 36.8 \% \\ 2.81 & 1.66 & 41.0 \% \\ 1.56 & 1.58 & -1.4 \% \\ 1.83 & 1.37 & 25.1 \% \\ 1.51 & 1.20 & 20.5 \% \\ 2.44 & 1.81 & 25.9 \% \\ & & \\ 2.08 & 1.49 & 28.5 \% \\ 2.51 & 2.22 & 11.6 \%\end{array}$

$1.01 \quad 1.06$

$-5.2 \%$

$0.99 \quad 0.97$

$1.8 \%$

$0.91 \quad 0.90$

$0.9 \%$

$0.87 \quad 0.97$

$-10.8 \%$

$0.99 \quad 1.07$

$-7.6 \%$

$\begin{array}{ll}0.97 & 0.94\end{array}$

$2.9 \%$

$0.76 \quad 0.68 \quad 10.8 \%$

$\begin{array}{lll}0.95 & 0.94 & 1.8 \%\end{array}$

$0.84 \quad 0.81 \quad 3.8 \%$ 
Figure 2. Relative Contributions to World High-impact Levels By the U.S. (red), the EU (blue), and the RW (green) According to the $\beta=2$ Member of the FGT Family of High-impact Indicators When the CCL $=80$ th and 95th Percentiles. Relative Contributions to the Top 5\% of Highly Cited Articles Appear As Black Horizontal Lines
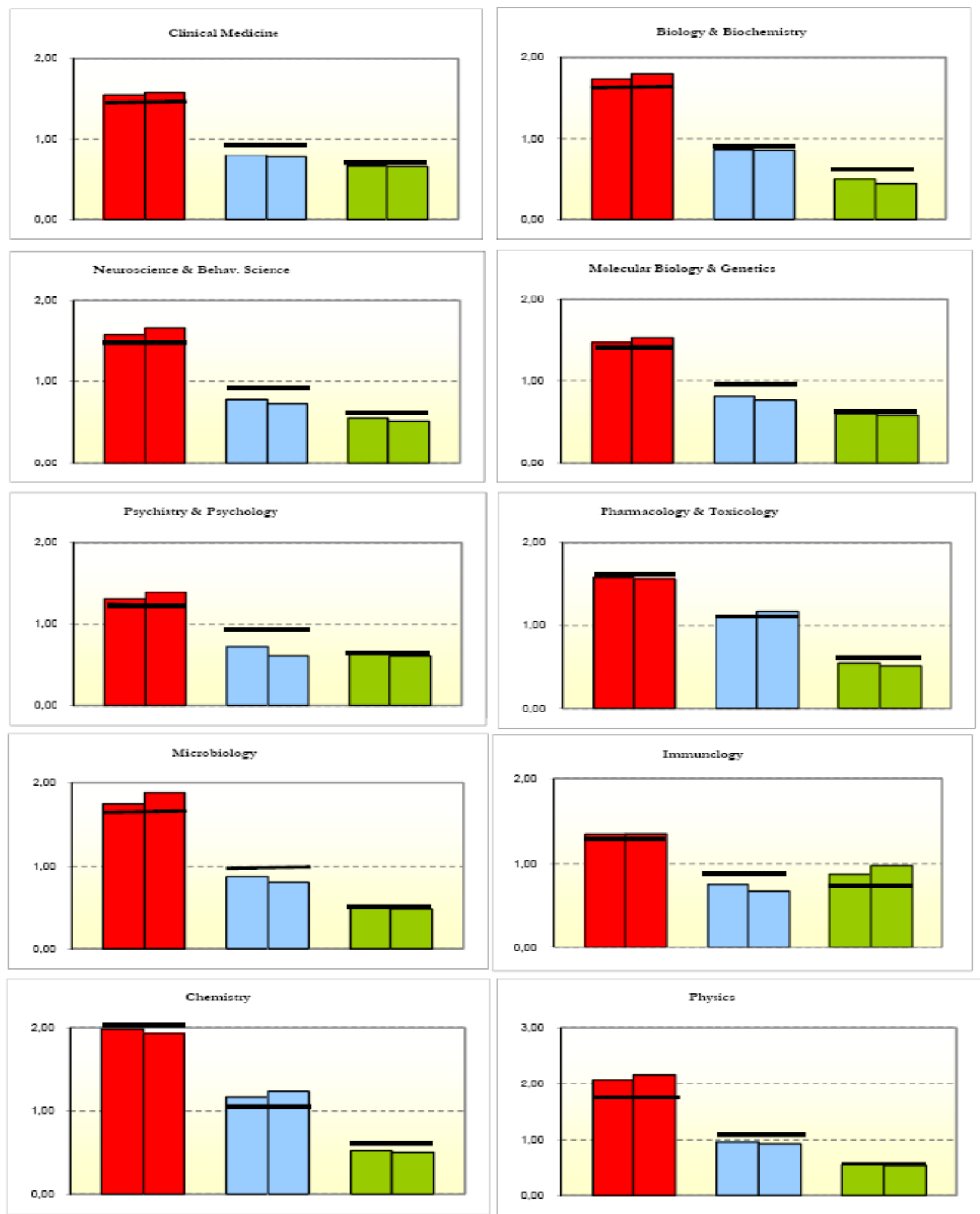
Figure 2. Relative Contributions to World High-impact Levels By the U.S. (red), the EU (blue), and the RW (green) According to the $\beta=2$ Member of the FGT Family of High-impact Indicators When the CCL $=80$ th and 95th Percentiles. Relative Contributions to the Top 5\% of Highly Cited Articles Appear As Black Horizontal Lines
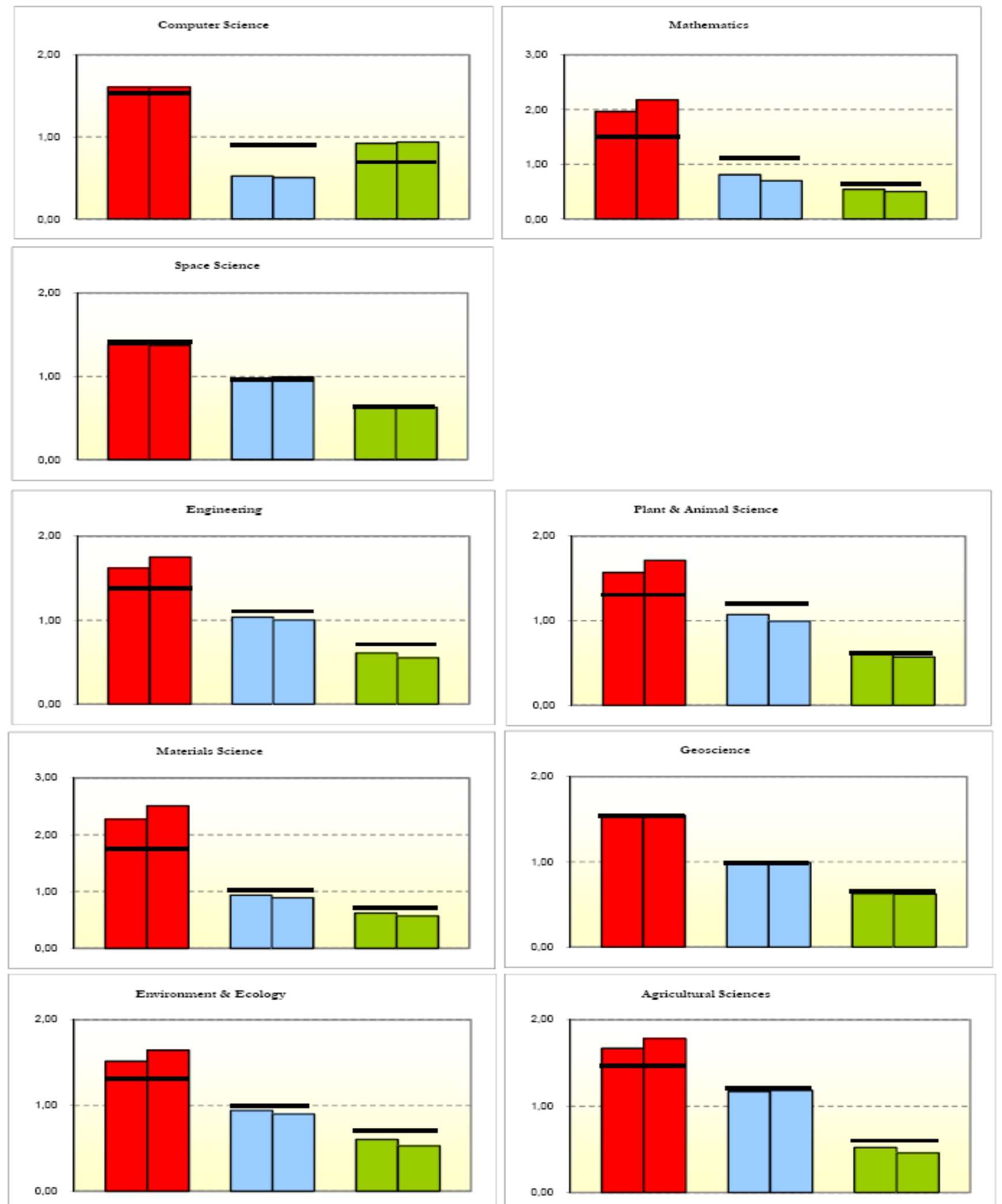

Figure 2. Relative Contributions to World High-impact Levels By the U.S. (red), the EU (blue), and the RW (green) According to the $\beta=2$ Member of the FGT Family of High-impact Indicators When the CCL $=80$ th 
and 95th Percentiles. Relative Contributions to the Top 5\% of Highly Cited Articles Appear As Black Horizontal Lines
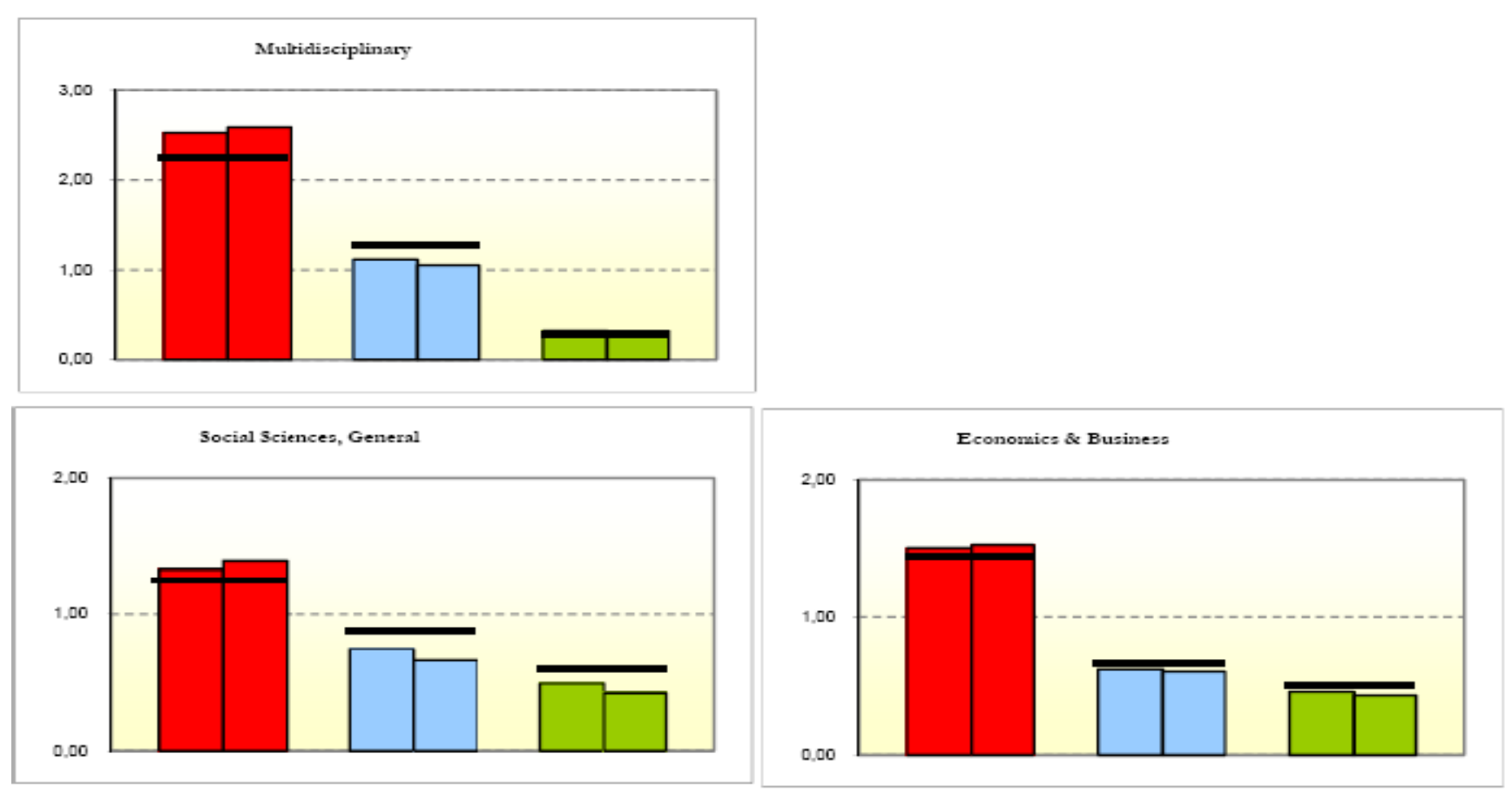
Figure 3. Relative Contribution to World Low-impact Levels By the U.S. (red), the EU (blue), and the RW (green) According to the $L_{2}$ Low-impact Indicator When the critical citation line is equal to the $80^{\text {th }}$ and $95^{\text {th }}$ Percentiles. Relative Contributions to the Percentage of Uncited Articles Appear As Black Horizontal Line
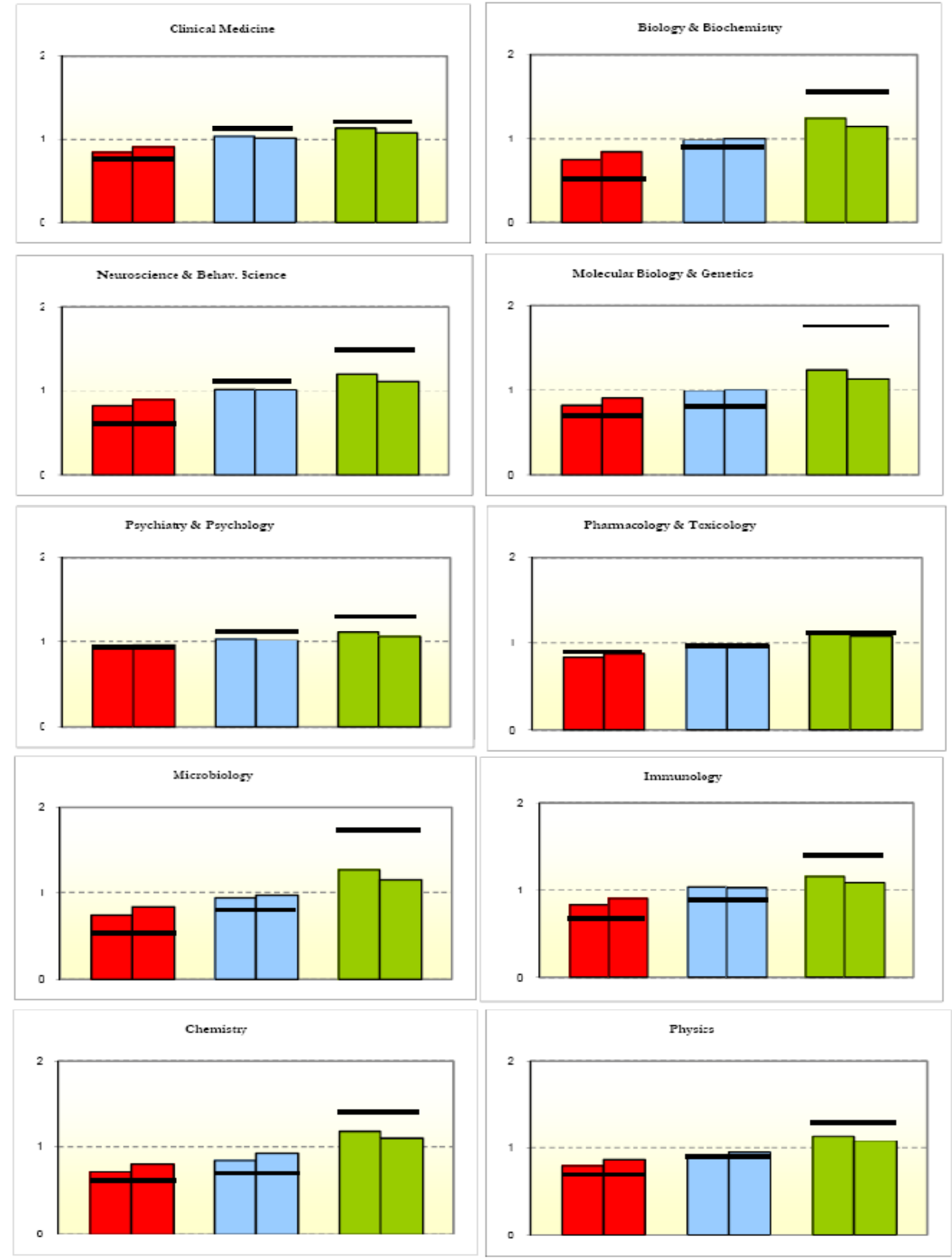
Figure 3. Relative Contribution to World Low-impact Levels By the U.S. (red), the EU (blue), and the RW (green) According to the $L_{2}$ Low-impact Indicator When the critical citation line is equal to the $80^{\text {th }}$ and $95^{\text {th }}$ Percentiles. Relative Contributions to the Percentage of Uncited Articles Appear As Black Horizontal Lines
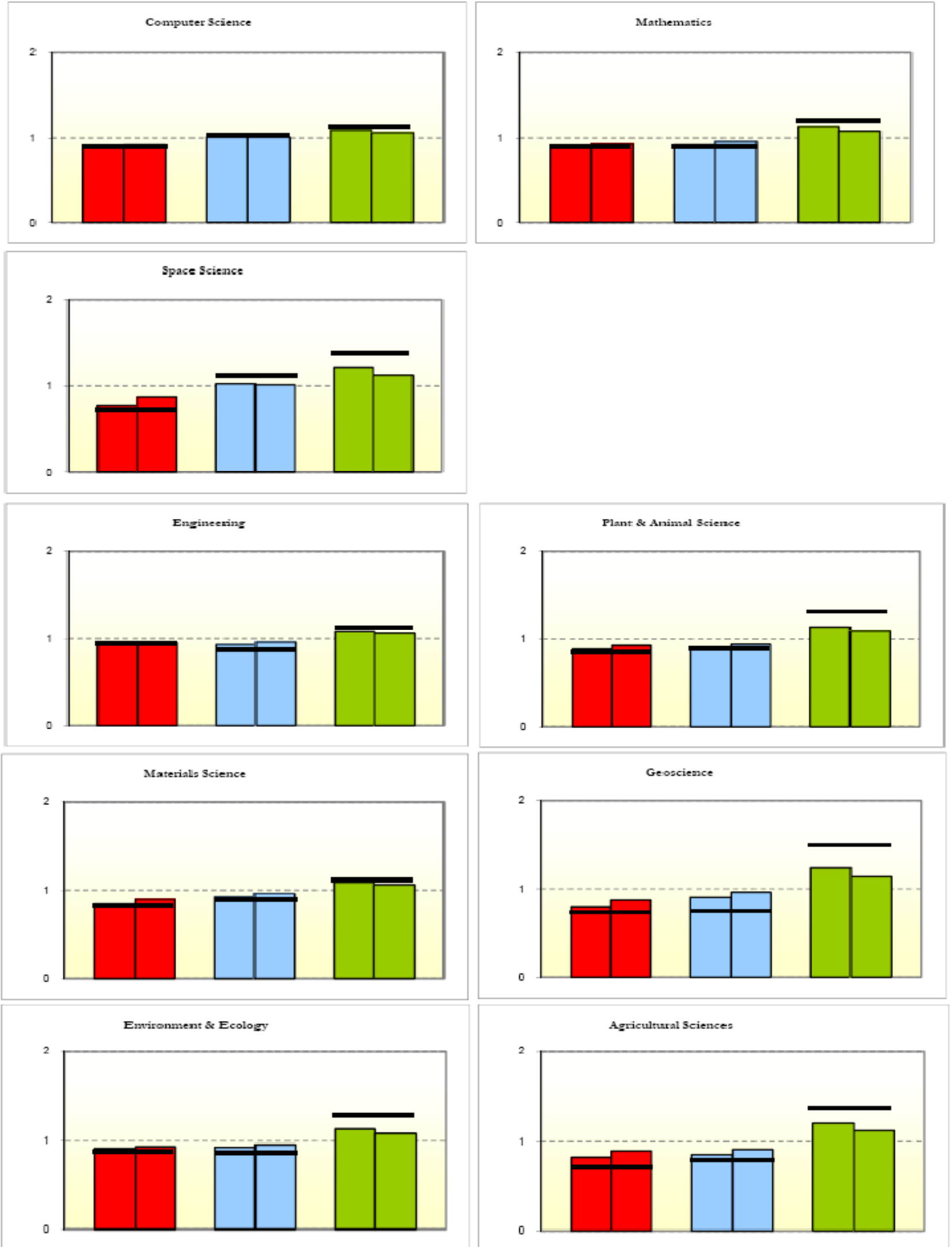
Figure 3. Relative Contribution to World Low-impact Levels By the U.S. (red), the EU (blue), and the RW (green) According to the $L_{2}$ Low-impact Indicator When the critical citation line is equal to the $80^{\text {th }}$ and $95^{\text {th }}$ Percentiles. Relative Contributions to the Percentage of Uncited Articles Appear As Black Horizontal Lines
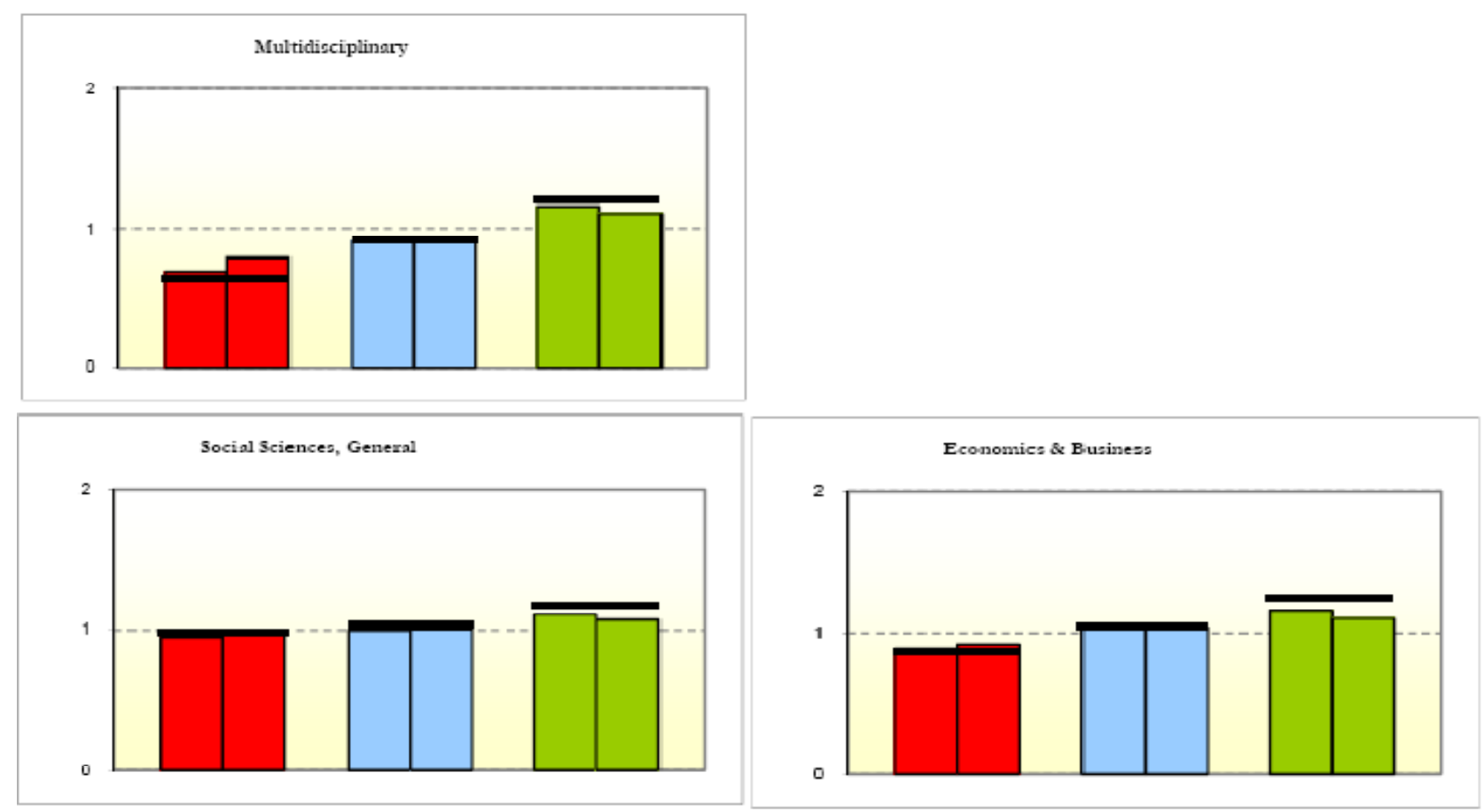